\title{
HOMOGENEOUS DIFFERENCE SCHEMES FOR NON-LINEAR PARABOLIC EQUATIONS*
}

\author{
A. A. SAMARSKII \\ Moscow \\ (Received 30 September 1961)
}

WE SHALL consider homogeneous difference schemes of "through" computation (see [1]-[3]) for the non-linear parabolic equation

$$
\mathscr{P} u=\frac{\partial}{\partial x}\left(k(x, t) \frac{\partial u}{\partial x}\right)+f\left(x, t, u, \frac{\partial u}{\partial x}, \frac{\partial u}{\partial t}\right)=0
$$

with a discontinuous "heat-conduction coefficient" $k(x, t)$.

Attention is devoted chiefly to determining the order of accuracy of the sixpoint schemes $\mathscr{P}_{h \tau}^{\alpha}$ (see [3]) for the third boundary problem in a bounded region

$$
\bar{д}(0 \leqslant x \leqslant 1,0 \leqslant t \leqslant T) .
$$

The investigation is carried out directly for a wide class of homogeneous difference schemes (see $\S 1.4$ ), given in terms of pattern functionals of the type indicated in [1] and [3]. The functionals satisfy conditions which ensure a second order of approximation (with respect to $x$ ) of the scheme. Similar difference schemes (in the case of the first boundary problem) for the linear heat-conduction equation when

$$
f\left(x, t, u, \frac{\partial u}{\partial x}, \frac{\partial u}{\partial t}\right)=f(x, t)-q(x, t) u-c(x, t) \frac{\partial u}{\partial t}
$$

were studied in [3].

Difference boundary conditions of the third kind, with the same order of approximation as the scheme in the class of solutions of the equations $\mathscr{P} u=0$ are formulated in $\S 2$.

The problem of the accuracy of the difference problem obtained can be reduced to estimating the solution $z$ of a linear difference equation with linear difference boundary conditions and zero initial condition by using the functions $\psi, \nu_{1}$ and $\nu_{2}$, where $\psi$ is the approximation error of the scheme, $\nu_{1}$ and $\nu_{2}$ are the approximation errors of the boundary conditions in the class of solutions of the equation $\mathscr{P}_{u}=0$. A priori estimates are used to evaluate the solution of this problem. These are generalized from the estimates [4] and [5] to a more general equation and more general conditions.

The so-called "fixed discontinuities" of the coefficient $k(x, t)$, i.e. discontinuities on the finite number of straight lines $x=\eta_{v}=$ const., parallel to the $t$-axis in the plane $(x, t)$, are considered in this paper. By separating the error connected with

* Zh. vych. mat. 2: No. 1, 25 56, 1962. 
the approximation error of the boundary conditions it can be shown that the third boundary problem has the same order of accuracy as the first (see $\S 3$ ).

The principal result has been formulated as Theorem 4 in $\S 3$. It has been proved that any scheme $\mathscr{P P}_{h \tau}^{\alpha}$ of the initial family (see $\S 1.4$ ) converges uniformly for $0.5 \leqslant$ $\leqslant \alpha \leqslant 1$, when the intervals of the difference set $h=\Delta x$ and $\tau=\Delta t$ tend to zero independently. The order of accuracy in the class of discontinuous coefficients is also evaluated. Results for the explicit schemes $(\alpha=0)$ are not given because they can be formulated by analogy with the case of the linear equation (3).

As has been pointed out several times (see [1], [3], [5]), the difficulty in investigating uniform convergence in the case of the discontinuous functions $k(x, t)$ and $f(x, t, u, p, q)$ lies in the fact that the difference scheme does not approximate to a differential operator near the line of discontinuity of the coefficient $k(x, t)$.

For six-point schemes convergences can be proved only by using an improved a priori estimate (Theorem 2) which uses the norms $\|\psi\|_{5}$ and $\|\psi\|_{5 *}$ of a special type for an integral estimate of the approximation error $\psi$.

Although uniform nets are considered, the principal results and, in particular, Theorem 4 are applicable to difference schemes on non-uniform nets also. This question has been treated separately.

The method of investigation adopted can be used to prove Theorem 4 for more general boundary conditions, including non-linear ones also. $\S 1$ and 3 remain practically unchanged in that case.

It should be noted that there are a number of papers (for example [6]-[11]) devoted to the investigation of difference schemes of a partial type for non-linear and quasi-linear parabolic equations (1) in the class of smooth functions $k(x, t)$ and $f(x, t, u, p, q)$. In most of the papers either average convergence or uniform convergence has been studied using the principle of the maximum (involving boundedness for $\tau / h^{2}$ ) for partial cases of eqn. (1). The method closest to ours is the one described in paper [10], in which the uniform convergence of a scheme for the case of boundary conditions of the first kind and the continuous functions $k(x, t)$ and $f(x, t, u, p, q)$ was proved. The results given there follow from Theorem 3 of our paper. The a priori estimates used in [10] are unsuitable for proving convergences in the class of discontinuous coefficients.

\section{\$1. HOMOGENEOUS DIFFERENCE SCHEMES FOR THE NON-LINEAR PARABOLIC EQUATION}

\section{Formulation of the problem}

In the rectangle $\bar{д}=(0 \leqslant x \leqslant 1,0 \leqslant t \leqslant T)$ let us consider the finite number of non-intersecting differentiable curves $\Gamma_{v}\left(v=0,1, \ldots, v_{0}+1\right)$ taken in pairs, given by the equations $x=\eta_{\nu}(t)$ in the segment $0 \leqslant t \leqslant T$. We re-number these curves in such a way that $\eta_{v_{1}}(t)<\eta_{v_{2}}(t)$ when $v_{1}<v_{2}$, and put $\eta_{0}(t)=0, \eta_{v_{0}+1}$ $(t)=1 . \Delta_{v}$ and $\not$ denote the regions 


$$
\left.\Delta_{v}=\left(\eta_{v}(t)<x<\eta_{v+1}(t)\right) 0<t \leqslant T\right), \quad 0 \leqslant v \leqslant \nu_{0}, \quad \bar{Z}=\sum_{v=0}^{v_{0}} \Delta_{v} .
$$

The initial problems is formulated as follows:

Find a solution $u(x, t)$ continuous in $\bar{\not}$ of the equation

$$
\partial u=\frac{\partial}{\partial x}\left(k(x, t) \frac{\partial u}{\partial x}\right)+f_{1}\left(x, t, u, \frac{\partial u}{\partial x}, \frac{\partial u}{\partial t}\right)=0 \text { in } \not,
$$

satisfying the boundary conditions

$$
\begin{aligned}
& k \frac{\partial u}{\partial x}-\sigma_{1}(t) u=u_{1}(t), \quad x=0 \\
& k \frac{\partial u}{\partial x}+\sigma_{2}(t) u=u_{2}(t), \quad x=1,
\end{aligned}
$$

and the initial condition

$$
u(x, 0)=u_{0}(x) .
$$

The coefficient $k(x, t)$ has discontinuities of the first kind on the curves $\Gamma_{\nu}(v$ $=1,2 \ldots, v_{0}$ ). The continuity conditions (matching on the left and right of the function $u(x, t)$ and $\left.k \frac{\partial u}{\partial x}\right)$ are satisfied on each of these curves:

$$
\left[u_{\nu}=0, \quad\left[k \frac{\partial u}{\partial x}\right]_{v}=0 \quad \text { for } x=\eta_{\nu}(t), \quad 0 \leqslant t \leqslant T,\right.
$$

where $[u]_{v}=u_{\Pi, v}-u_{\pi, v}, u_{\pi, v}=u\left(\eta_{v}(t)+0, t\right), u_{\pi, v}=u\left(\eta_{v}(t)-0, t\right)$ etc.

The function $f_{1}(x, t, u, p, q)$ for the variables $(x, t)$ has discontinuities of the third kind on the curves $\Gamma_{\nu}, v=1,2, \ldots, v_{0}$, and its derivatives with respect to $u, p$ and $q$ are bounded, or more precisely,

$$
0<m \leqslant-\frac{\partial f_{1}}{\partial q} \leqslant M, \quad\left|\frac{\partial f_{1}}{\partial u}\right| \leqslant M, \quad\left|\frac{\partial f_{1}}{\partial p}\right| \leqslant M,
$$

where $m$ and $M$ are positive constants.

The coefficients $k(x, t)$ and $\sigma_{1}(t), \sigma_{2}(t)$ of the problem satisfy the conditions

$$
0<m \leqslant k(x, t) \leqslant M, \quad \sigma_{1}(t) \geqslant 0, \quad \sigma_{2}(t) \geqslant 0, \quad \sigma_{1}(t)+\sigma_{2} \geqslant m>0
$$

The condition $\sigma_{1}(t)+\sigma_{2}(t) \geqslant m>0$ signifies that the case when $\sigma_{1}(t)=0$ and $\sigma_{2}(t)=0$ simultaneously (second boundary condition) is not considered. Without any loss in generality we put

$$
f_{1}\left(x, t, u \cdot \frac{\partial u}{\partial x}, \frac{\partial u}{\partial t}\right)=f\left(x, t, u, 2 k(x, t) \frac{\partial u}{\partial x}, \frac{\partial u}{\partial t}\right),
$$

i.e. henceforth we shall consider equation (1) in the form

$$
\mathcal{P} u=\frac{\partial}{\partial x}\left(k(x, t) \frac{\partial u}{\partial x}\right)+f\left(x, t, u, 2 k \frac{\partial u}{\partial x}, \frac{\partial u}{\partial t}\right)=0,
$$

This substitution is convenient because the argument $k \frac{\partial u}{\partial x}$ is continuous because 
of the condition (5) on the lines $\Gamma$, of discontinuity of the coefficient $k(x, t)$. Since $k(x, t) \geqslant m>0$, the conditions (6) for the function $f(x, t, u, p, q)$ will be satisfied.

We shall call the problem defined by the conditions $\left(1^{\prime}\right),(2)-(7)$, the problem (I) It will be noted that the equation

$$
\frac{\partial}{\partial x}\left(k(x, t) \mu^{*}(u) \frac{\partial u}{\partial x}\right)+f_{1}\left(x, t, u, \frac{\partial u}{\partial x}, \frac{\partial u}{\partial t}\right)=0,
$$

is reduced to equation (1) if the new function

$$
\bar{u}=\mu(u)=\int_{0}^{u} \mu^{*}(u) \mathrm{d} u .
$$

is introduced.

The existence and uniqueness of the solution of boundary problems for a parabolic linear equation of the type

$$
\frac{\partial}{\partial x}\left(k(x, t) \frac{\partial u}{\partial x}\right)+r(x, t) \frac{\partial u}{\partial x}-q(x, t) u+f(x, t)-c(x, t) \frac{\partial u}{\partial t}=0
$$

with discontinuous coefficients was studied in [12]-[15]. A large number of papers have been devoted to the solubility of the quasi-linear equation

$$
\frac{\partial}{\partial x}\left(k(x, t) \frac{\partial u}{\partial x}\right)+r(x, t, u) \frac{\partial u}{\partial x}+f(x, t, u)-c(x, t, u) \frac{\partial u}{\partial t}=0
$$

with smooth coefficients.

We shall assume below that a solution of the problem (I) exists and has the differential properties necessary for our discussion, leaving aside the question of conditions ensuring these properties. Only those requirements for coefficients of the problem are formulated which are used directly in proving theorems on the accuracy of the difference schemes studied.

Note 1 . The derivative $\frac{\partial u}{\partial t}$ on the lines $\Gamma$, of discontinuity of the "heat-conduction coefficient" $k(x, t)$ undergoes a discontinuity equal to

$$
\left[\frac{\partial u}{\partial t}\right]_{\nu}=-\left[\frac{\partial u}{\partial x}\right]_{\nu} \eta_{\nu}^{\prime}(t), \quad x=\eta_{\nu}(t)
$$

The equation (13) follows from the identity

$$
\frac{\mathrm{d} u\left(\eta_{\nu}(t), t\right)}{\mathrm{d} t}\left(\frac{\partial u}{\partial t}\right)_{\pi, v}+\left(\frac{\partial u}{\partial x}\right)_{\pi, \nu} \eta_{p}^{\prime}(t)=\left(\frac{\partial u}{\partial t}\right)_{\mathrm{n}, \boldsymbol{v}}+\left(\frac{\partial u}{\partial x}\right)_{\mathrm{n}, \boldsymbol{v}} \eta_{\nu}^{\prime}(t)
$$

It can be seen from (13) that the derivative $\partial u / \partial t$ is continuous along $\Gamma$, only when $\eta_{\nu}^{\prime}(t)=0$ i.e. on these straight lines $\eta_{y}=$ const. (in this case we say that $k(x, t)$ has a fixed discontinuity).

NoTE 2. If the new function

$$
v=u \exp \left(\int_{0}^{x}(r / 2 k) \mathrm{d} \xi\right),
$$

is introduced, the linear equation (11) is transformed into a linear equation for $v$ not containing a term with the first derivative with respect to $x(r=0)$. But with this transformation the second continuity condition becomes more complex.

$$
\left[k \frac{\partial v}{\partial x}-r v\right]_{v}=0 \quad x=\eta_{\nu}(t)
$$




\section{Integro-interpolation method}

The integro-interpolation method (IIM) (see [1], [2]) can be used to obtain difference equations corresponding to the differential equation (1). Since physical processes are usually characterized by integral equations expressing a certain law of conservation (for example, the amount of heat, momentum etc.), in writing difference equations describing the process approximately it is natural to start with integral balance equations written for a certain volume in the neighbourhood of a nodal point of the difference net. The integrals of the function and its derivatives occurring in the equation and taken over the surface of the volume considered are replaced by difference expressions by making additional assumptions regarding the behaviour of the function in the neighbourhood of the nodal point. As a result we obtain difference equations the form of which depends on the nature of the local interpolation used. The choice of interpolation is subjected to the requirements of stability, accuracy and simplicity (for the same order of accuracy) which the required difference scheme must satisfy. As an illustration of the method we take the heat-conduction equation

$$
\frac{\partial u}{\partial t}=\frac{\partial}{\partial x}\left(k(x, t) \frac{\partial u}{\partial x}\right)+f(x, t) .
$$

We consider in the rectangle $(0 \leqslant x \leqslant 1,0 \leqslant t \leqslant T)$ a uniform net $\bar{\Omega}$, i.e. a set of points $\left(x_{i}, t_{j}\right)$ with the coordinates $x_{i}=i h, i=0,1, \ldots, N(h=1 / N) ; t_{j}=j \tau$, $j=0,1, \ldots, L(\tau=T / L)$. The heat-balance equation for the rectangle $S_{i j}$ $=\left(x_{i-\frac{1}{2}} \leqslant x \leqslant x_{i+\frac{1}{2}}, t_{j-1} \leqslant t \leqslant t_{j}\right), x_{i-\frac{1}{2}}=x_{i}-0 \cdot 5 h$ is:

$$
\begin{array}{r}
\int_{x_{i-\frac{1}{2}}}^{x_{i+\frac{1}{2}}}\left[u\left(x, t_{j}\right)-u\left(x, t_{j-1}\right)\right] \mathrm{d} x+\int_{t_{j-1}}^{t_{j}}\left[W\left(x_{i+\frac{1}{2}}, t\right)-W\left(x_{i-\frac{1}{2}}, t\right)\right] \mathrm{d} t \\
=\iint_{S_{i j}} f(x, t) \mathrm{d} x \mathrm{~d} t,
\end{array}
$$

where $W=-\mathrm{k} \frac{\partial u}{\partial x}$ is the thermal flow.

We shall consider that the values of $u$ are taken at the points $\left(x_{i}, t_{j}\right)$ of the net and the flow $W$ refers to the point $\left(x_{i-\frac{1}{2}}, t_{j}\right)$.

Suppose, for example, that $W=W_{i-\frac{1}{2}}=$ const. in the interval $\left(x_{i-1}, x_{i}\right)$. Then the expression $W=-k \frac{\partial u}{\partial x}$ will be replaced by the approximate relation

$$
u_{i-1}-u_{i}=\int_{x_{i-1}}^{x_{i}}(W / k) \mathrm{d} x \approx W_{i-\frac{1}{2}} \int_{x_{i-1}}^{x_{i}} \frac{\mathrm{d} x}{k},
$$

i.e.

$$
W_{i-\frac{1}{2}}=a_{i} \frac{u_{i-1}-u_{i}}{h}, \quad a_{i}=\left[\frac{1}{h} \int_{x_{i-1}}^{x_{i}} \frac{\mathrm{d} x}{k}\right]^{-1} .
$$


To calculate the first integral in the balance equation (15) we suppose that $u$ $=u_{i}=$ const. in the interval $\left(x_{i-\frac{1}{2}}, x_{i+\frac{1}{2}}\right)$, so that

$$
\frac{1}{h} \int_{x_{i-\frac{1}{2}}}^{x_{i+\frac{1}{2}}} u \mathrm{~d} x=u_{i} .
$$

It can be easily seen that the assumptions $u=$ const. when $x_{i-\frac{1}{2}}<x<x_{i+\frac{1}{2}}$ and $W=$ const. when $x_{i-1}<x<x_{i}$ do not in general agree with one another. But the assumption $W=$ const. when $x_{i-1}<x<x_{1}$, if consistently carried out, leads to a more complicated scheme without increasing the accuracy.

Other interpolations of the required functions for deriving a difference expression for the flow are also possible.

If the flow is to be expressed in terms of the value of $u$ at two nodal points, then from the equation

$$
u_{i-1}-u_{i}=\int_{x_{i-1}}^{x_{i}} \frac{W}{k} \mathrm{~d} x
$$

it follows that all formulae obtained for $W_{i-\frac{1}{2}}$ will have the form

$$
W_{i-\frac{1}{2}}=a_{i}^{(h, k)}\left(u_{i-1}-u_{i}\right) / h,
$$

where $a_{i}^{(h, k)}$ is a coefficient depending in general on $h$ and on a function $k(x, t)$ in the interval $\left(x_{i-1}, x_{i}\right)$, i.e. it is a functional of the coefficient $k$.

If the integrals in $t$ in equation (15) are replaced by, for example, the formula

$$
\int_{t_{j-1}}^{t_{j}} W \mathrm{~d} t=\alpha W^{j}+(1-\alpha) W^{j-1}, \quad 0 \leqslant \alpha \leqslant 1,
$$

we obtain the difference equation

$$
\begin{aligned}
& \frac{y_{i}^{j}-y_{i}^{j-1}}{\tau}=\alpha \frac{W_{i-\frac{1}{2}}^{j}-W_{i+\frac{1}{2}}^{j}}{h}+(1-\alpha) \frac{W_{i-\frac{1}{2}}^{j-1}-W_{i+\frac{1}{2}}^{j-1}}{h}+F_{i}^{j} ; \\
& W_{i-\frac{1}{2}}=a_{i}^{(h, k} \frac{y_{i-1}-y_{i}}{h}, \quad F_{i}^{j}=\frac{1}{h \tau} \int_{t_{j-1}}^{t_{j}} \int_{i-\frac{1}{2}}^{x_{i+\frac{1}{2}}} j(x, t) \mathrm{d} x \mathrm{~d} t .
\end{aligned}
$$

The balance method (IIM) leads to convergent schemes in the class of discontinuous coefficients, as was shown in [1] for the stationary equation of heat conduction. In this paper we shall show that this is also true for a parabolic equation.

\section{Notations}

Let $\bar{\Omega}\left(x_{i}=i h, t_{j}=j \tau, i=0,1, \ldots N, j=0,1, \ldots, L, h=1 / N, \tau=T / L\right)$ be a difference net, $\bar{\Omega}$ the set of its internal points $\left(x_{i}, t_{j}\right)$ (where $1 \leqslant i \leqslant N-1,1 \leqslant$ $\leqslant j \leqslant L), \omega_{h}\left(x_{i}=i h, 0 \leqslant i \leqslant N\right)$ is a $x$-net. 
A function $y_{i}^{j}$ given on the net $\bar{\Omega}$ or part of it will be called a net function. Instead of $y_{i}^{j}$ we shall simply write $y$ or $y(x, t)$; the dependence of the net function on the intervals $h$ and $\tau$ of the net also will not be indicated. Further, we shall use the notations

$$
\begin{array}{cl}
\check{y}=y(x, t-\tau), & y^{(+1)}=y(x+h, t), \quad y^{(-1)}=y(x-h, t), \\
y_{\bar{x}}=\left(y-y^{(-1)}\right) / h, & y_{x}=\left(y^{(+1)}-y\right) / h=y_{\bar{x}}^{(+1)}, \quad y_{\bar{t}}=(y-\check{y}) / \tau .
\end{array}
$$

Hence

$$
\frac{1}{h^{2}}\left[a_{i+1}^{j}\left(y_{i+1}^{j}-y_{i}^{j}\right)-a_{i}^{j}\left(y_{i}^{j}-y_{i-1}^{j}\right)\right]=\left(a y_{-}\right)_{x} .
$$

We shall use the following notations for sums over the net $\omega_{k}$ or part of it

$$
\begin{aligned}
(y, v)=\sum_{i=1}^{N-1} y_{i} v_{i} h ; \quad(y, v] & =\sum_{i=1}^{N} y_{i} v_{i} h ; \quad[y, v)=\sum_{i=0}^{N-1} y_{i} v_{i} h ; \\
{[y, v] } & =\sum_{i=0}^{N} y_{i}, v, h .
\end{aligned}
$$

We shall use the norm

$$
\|\left. z\right|_{0}=\max _{(x)}|z|,
$$

where the maximum value is taken from all points of the net $\omega_{h}$ in which the net function $z$ is given.

Depending on where the function $\psi$ is given, we use one of the following definitions for the norms:

$$
\|\psi\|_{\sigma}=\left(1,|\psi|^{\sigma}\right)^{\frac{1}{\sigma}}, \quad\|\psi\|_{\sigma}=\left(1,|\psi|^{\mid \sigma^{\frac{1}{\sigma}}}, \quad \mid \psi \psi \|_{\sigma}=\left[1,|\psi|^{\sigma}\right]^{\frac{1}{\sigma}}, \quad \sigma=1,2,\right.
$$

without specifying this in cases where there is no possibility of confusion. Thus, for example, if the function $z$ is given on the entire net $\omega_{h}$, its "difference derivative" $z_{x}$ is defined at the points $x=h, 2 h, \ldots, N h=1$; therefore

$$
\left\|z_{\bar{x}}\right\|_{2}=\left(1, z_{\bar{x}}^{2} 1^{\frac{1}{2}}, \quad \text { where } z_{\bar{x}}^{2}-\left(z_{\bar{x}}\right)^{2} .\right.
$$

In our previous papers (see [4], [5]) we introduced the norms

$$
\left.\begin{array}{l}
\|\psi\|_{3}=\|\mu\|_{2}, \quad \mu(x)=\sum_{x^{\prime}=h}^{x^{\prime}=x} h \psi\left(x^{\prime}\right), \\
\left.\quad\|\psi\|_{4}=\|\psi\|_{3}+\mid \psi, 1\right)\left|, \quad\|\psi\|_{4^{*}}=\|\psi\|_{3^{*}}+\right|(\psi, 1) \mid .
\end{array}\right\}
$$

The positive constants independent of the nets (i.e. of $h$ and $\tau$ ) will be denoted by $M$ and $m . \varrho(\delta)$ will everywhere denote an expression uniformly tending to zero when $\delta \rightarrow 0$, so that $|\varrho(\delta)|<\varrho_{0}(\delta)$, where $\varrho_{0}(\delta)$ depends only on $\delta$ and $\varrho_{0}(\delta) \rightarrow 0$ when $\delta \rightarrow 0 ; O\left(\delta^{m}\right)(m>0)$ is an expression with the order of magnitude $\delta^{m}$ when $\delta \rightarrow 0$, so that $\left|0\left(\delta^{m}\right)\right| \leqslant M \delta^{m}$ 
When no confusion can arise we shall write

$$
u^{\prime}=\frac{\partial u}{\partial x}, \quad \dot{u}=\frac{\partial u}{\partial t}
$$

In referring to formulae of another section we shall use two numbers, for example (1.20) is the formula $(20)$ of $\S 1$.

\section{Initial family of homogeneous difference schemes}

The integro-interpolation method described in section 2 is useful in selecting the initial class of schemes for differential equation (1). As we have seen in the example of the linear heat-conduction equation, this method gives six-point schemes, the space part of which is a self-conjugate or conservative scheme of the type $\left(a y_{\bar{x}}\right)_{x}+\varphi$ and the coefficients $a$ and $\varphi$ are functionals (depending on the nature of interpolation) of the coefficients $k$ and $f$ of the differential equation.

On the other hand, it was shown in [1] in the example of the stationary heatconduction equation $\left(k u^{\prime}\right)^{\prime}+f=0$ that only the conservative schemes $\left(a y_{\bar{x}}\right)_{x}+\varphi$ converge in the class of discontinuous coefficients. We shall bear these two points in mind in choosing the initial family of difference schemes for non-linear parabolic equations.

Let us consider the differential equation

$$
\mathscr{P} u=\frac{\partial}{\partial x}\left(k(x, t) \frac{\partial u}{\partial x}\right)+f\left(x, t, u, 2 k \frac{\partial u}{\partial x}, \frac{\partial u}{\partial t}\right)=0
$$

and the corresponding six-point homogeneous difference scheme

$$
\begin{aligned}
\mathscr{P}_{h t}^{\alpha} y=\left(a y_{\bar{x}}\right)_{x}^{(\alpha)}+\alpha \varphi\left(x, t, y, a^{(+1)} y_{x}+a y_{\bar{x}}, y_{\bar{t}}\right)+ \\
\quad+(1-\alpha) \varphi\left(x, \check{t}, \check{y}, \check{a}^{(+1)} \check{y}_{x}+\check{a ̆}_{\bar{x}}, y_{\bar{t}}\right)=0 .
\end{aligned}
$$

The superscript $\alpha$ indicates that the expression is summed up over the rows $t$ and $t-\tau$ with the weight factors $\alpha$ and $(1-\alpha)$

$$
v^{(\alpha)}=\alpha v+(1-\alpha) \grave{v}
$$

where $\alpha$ is a numerical parameter, $0 \leqslant \alpha \leqslant 1$.

The net functions $a(x, t)$ and $\varphi(x, t, u, p, q)$ are calculated with pattern functionals (see [1], [3])

$$
A^{h}[\mu(s)] \quad(-1 \leqslant s \leqslant 0) \quad \text { and } \quad F^{h}[\mu(s)] \quad(-0.5 \leqslant s \leqslant 0.5),
$$

defined in the class of piecewise continuous functions $Q^{(0)}\left(\mu(s) \in Q^{(0)}\right)$ and depending in general on the parameter $h$ :

$$
\begin{aligned}
& a=A^{h}[k(x+s h, t)], \\
& \quad \varphi=\varphi\left(x, t, y, a^{(+1)} y_{x}+a y_{\bar{x}}, y_{\bar{t}}\right)=F^{h}\left[f\left(x+s h, t, y, a^{(+1)} y_{x}+a y_{\bar{x}}, y_{\bar{t}}\right)\right] .
\end{aligned}
$$

The dependence of $a$ and $\varphi$ on $h$ will not be indicated explicitly. 
For the linear equation

$$
\mathscr{P} u=\frac{\partial}{\partial x}\left(k(x, t) \frac{\partial u}{\partial x}\right)-q(x, t) u+r(x, t) \frac{\partial u}{\partial x}-c(x, t) \frac{\partial u}{\partial t}=0
$$

the difference scheme (25) assumes the form

$$
\mathscr{P}_{h x}^{\alpha} y=\left[\left(a y_{\bar{x}}\right)_{x}-d y+b\left(a^{(+1)} y_{x}+a y_{\bar{x}}\right)+\varphi\right]^{(\alpha)}-\varrho^{(\alpha)} y_{\bar{t}}=0 ;
$$

its coefficients are determined with the same pattern functionals (26) by the formulae:

$$
\begin{gathered}
a=A^{h}[k(x+s h, t)], \quad d=F^{h}[q(x+s h, t)], \quad b=F^{h}[\tilde{r}(x+s h, t)] \quad \tilde{r}=r / 2 k, \\
\varphi=F^{h}[f(x+s h, t)], \quad \varrho=F^{h}[c(x+s h, t)] .
\end{gathered}
$$

A class of homogeneous schemes for the linear equation (11) when $r(x, t) \equiv 0$ was introduced in paper [3]. The difference between those schemes and the ones considered here is that the coefficients $d$ and $\varrho$ were calculated independently of the coefficient $\varphi$ using the functionals $D^{h}[\mu(s)]$ and $R^{h}[\mu(s)](-0.5 \leqslant s \leqslant 0.5)$ respectively. The term $r(x, t) \frac{\partial u}{\partial x}$ in (11) can be approximated by using a simpler expression, for example

$$
F^{h}[r(x+s h, t)] \cdot 0 \cdot 5\left(y_{x}+y_{\bar{x}}\right) .
$$

But, as will be seen below, a higher order of accuracy in the class of discontinuous coefficients can be obtained by approximating with the expression $b\left(a^{(+1)} y_{x}+\right.$ $\left.+a y_{\bar{x}}\right)$.

For the same reason we take $2 k u^{\prime}$ (see section 2) and not $u^{\prime}$ as the argument of the function $f$ :

The class of homogeneous difference schemes $\mathscr{P}_{h r}^{x}$ is determined by choosing the class of pattern functionals $A^{h}$ and $F^{h}$ and the parameter $\alpha$. We shall assume that the $(\Phi)$ conditions are fulfilled:

$\left(\Phi_{1}\right)$ The functional of $A^{h}[\mu]$ is of the third rank and $F^{h}(\mu]$ of the second rank;

$\left(\Phi_{2}\right) F^{h}[\mu]$ is a linear functional;

$\left(\Phi_{3}\right)$ The pattern functionals $A^{h}[\mu]$ and $F^{h}[\mu]$ satisfy the necessary conditions for the second order of approximation (see [1], [3]).

We shall examine the six-point schemes (25) satisfying the conditions $(\Phi)$ as an initial family of schemes.

If the pattern functionals are canonical, i.e. are independent of $h$, the scheme is called canonical. Since non-canonical schemes are equivalent to their canonical part as shown in [1], [3], we shall deal with canonical schemes only. However, all the results are also valid for non-canonical schemes forming the initial family defined above.

Let us consider the properties of the canonical pattern functionals $A[\mu]$ and $F[\mu]$ in greater detail. Here the conditions $(\Phi)$ denote:

1) $A[\mu]$ is a linear homogeneous functional $(A[c \mu]=c A[\mu])$ having a differential of the third order, i.e. 


$$
A[\mu+\delta \cdot f]=A[\mu]+\delta \cdot A_{1}[\mu, f]+\delta^{2} A_{2}[\mu, f]+\delta^{3} A_{3}[\mu, f]+\delta^{3} \varrho(\delta),
$$

where $\varrho(\delta) \rightarrow 0$ when $\delta \rightarrow 0,|f|<M$;

2) $A[\mu]$ and $F[\mu]$ are non-decreasing functionals, i.e.

$$
A\left[\mu_{2}\right] \geqslant A\left[\mu_{1}\right], \quad F\left[\mu_{2}\right] \geqslant F\left[\mu_{1}\right] \quad \text { for } \mu_{2} \geqslant \mu_{1} ;
$$

3) $A[\mu]$ and $F[\mu]$ are normalized functionals, i.e.

$$
A[1]=F[1]=1 \text {; }
$$

4) $A[\mu]$ and $F[\mu]$ satisfy the necessary conditions of a second order approximation

where

$$
A_{1}[s]=-0.5, \quad F[s]=0,
$$

$$
A_{1}[s]=A_{1}[1, s] \text {. }
$$

It will be noted that schemes symmetrical with respect to change in the direction of the $x$-axis satisfy the conditions (27).

The difference scheme $\mathscr{P}_{h \tau}^{\alpha}$ belonging to the initial family, and having the pattern functionals (see [16])

$$
A[\mu(s)]=\left[\int_{-1}^{0} \frac{\mathrm{d} s}{\mu(s)}\right]^{-1}, \quad F[\mu(s)]=\int_{-0.5}^{0.5} \mu(s) \mathrm{d} s .
$$

so that

$$
a(x, t)=\left[\frac{1}{h} \int_{x-h}^{x} \frac{\mathrm{d} \xi}{k(\xi, t)}\right]^{-1}, \quad \varphi(x, t, y, p, q)=\frac{1}{h} \int_{x=0.5 h}^{x+0.5 h} f(\xi, t, y, p, q) \mathrm{d} \xi
$$

occupies a special position.

This scheme will be called the best canonical scheme because it gives the best accuracy in the class of discontinuous coefficients among the initial schemes.

In practice discrete schemes [1] are often used, the coefficients of which are calculated from the values of the coefficients of the differential equation at particular points. We shall give the pattern functionals for some of the discrete schemes:

$$
\begin{gathered}
A[\mu(s)]=\frac{2 \mu(0) \mu(-1)}{\mu(0)+\mu(-1)}, \quad A[\mu(s)]=0 \cdot 5[\mu(0)+\mu(-1)], \\
A[\mu(s)]=\mu(-0 \cdot 5), \\
F[\mu(s)]=\mu(0), \quad F[\mu(s)]=0.5[\mu(0.5)+\mu(-0.5)] \text { etc. }
\end{gathered}
$$

instead of scheme (25) another equivalent to it in accuracy can be considered

$$
\mathscr{P}_{h \tau}^{\alpha} y=\left(a y_{\bar{x}}\right)_{x}^{(\alpha)}+\varphi\left(x, t^{(\alpha)}, y^{(\alpha)},\left(a^{(+1)} y_{x}+a y_{\bar{x}}\right)^{(\alpha)}, y_{\bar{t}}\right)=0 \text {, }
$$

where $t^{(\alpha)}=\alpha t+(1-\alpha) \breve{t}, \check{t}=t-\tau$.

The difference equations $\mathscr{P}_{k x}^{\alpha} y=0$, where $\mathscr{P}_{k x}^{\alpha} y$ is determined by the expressions (25) or $\left(25_{1}\right)$, are non-linear with respect to the values of the required function $y(x, t)$ on the new row. For this reason its solution can be found only by iteration. The difference equations 


$$
\mathcal{P}_{h \tau}^{\alpha} y=\left(a y_{\bar{x}}\right)_{x}^{(\alpha)}+\varphi\left(x, \check{t}, \check{y}, \check{a}^{(+1)} \check{y}_{\bar{x}}+\check{a}^{2} \check{y}_{\bar{x}}, y_{\bar{t}}\right)=0
$$

are linear with respect to $y(x, t)$, if the function $f(x, t, u, p, q)$ depends linearly on $q$ :

$$
f(x, t, u, p, q)=f(x, t, u, p)+\bar{f}(x, t, u, p) q .
$$

In this case the difference equations $\left(25_{2}\right)$ can be solved by the non-iterative method of successive substitution (see $\$ 2.4$ ). But the difference scheme $(25)_{2}$ is only of the first order of the approximation with respect to $t$ for any $\alpha, 0 \leqslant \alpha \leqslant 1$.

It should be noted that all the results obtained below for the scheme (25) can be easily transferred to schemes $\left(25_{1}\right)$ and $\left(25_{2}\right)$.

The family of homogeneous schemes for the equation

$$
\rho u=\frac{\partial}{\partial x}\left[x(x, t, u) \frac{\partial u}{\partial x}\right]+f\left(x, t, u, 2 x \frac{\partial u}{\partial x}, \frac{\partial u}{\partial t}\right)=0
$$

with the heat-conduction coefficient $\varkappa$ depending on the temperature $u$ can be determined similarly In this case

$\mathcal{P}_{h \tau}^{n} y=\left(a y_{\bar{x}}\right)_{x}^{(\alpha)}+a \varphi\left(x, t, y, a^{(+1)} y_{x}+a y_{\bar{x}}, y_{\bar{t}}\right)+$

where

$$
+(1-\alpha) \varphi\left(x, \check{t}, \check{y}, \check{a}^{(+1)} \breve{y}_{x}+\check{a}_{\bar{y}} \grave{y}_{\bar{x}}, \mathrm{y}_{\bar{\imath}}\right)=0,
$$

$$
\begin{aligned}
& a=a\left(x, t, y^{*}\right)=A^{h}\left[x\left(x+s h, t, y^{*}\right)\right], \quad y^{*}=0.5(y+y(-1)), \\
& \varphi(x, t, u, p, q)=F^{h}[f(x+s h, t, u, p, q) .
\end{aligned}
$$

Here $A^{h}$ and $F^{h}$ are the functionals defined above.

\section{Equation for the solution error}

Let $y=y(x, t)$ be a solution of the difference equation (25) and $u=u(x, t)$ a solution of the differential equation (24). We shall show that the error $z=y-u$ satisfies a linear difference equation. To do this we form the difference

$$
\mathscr{P}_{h \tau}^{\alpha} y-(\mathscr{P} u)^{(\alpha)}=\left[\mathscr{P}_{h \tau}^{\alpha} y-\mathscr{P}_{h \tau}^{\alpha} u\right]+\Psi=0 \text { on } \Omega,
$$

where

$$
\Psi=\mathcal{P}_{h \tau}^{\alpha} u-(\mathcal{P} u)^{(\alpha)}
$$

Let us transform the expression

where

$$
\mathscr{P}_{h \tau}^{\alpha} y-\mathscr{P}_{h \tau}^{\alpha} u=\left(a z_{x}^{-}\right)_{x}^{(\alpha)}+\alpha B_{1}+(1-\alpha) B_{2},
$$

$$
\begin{aligned}
& B_{1}=\varphi\left(x, t, y, a^{(+1)} y_{x}+a y_{\bar{x}}, y_{\bar{t}}\right)-\varphi\left(x, t, u, a^{(+1)} u_{x}+a u_{\bar{x}}, u_{\bar{t}}\right), \\
& B_{2}=\varphi\left(x, \check{t}, \check{y}, \check{a}^{(+1)} \check{y}_{x}+\check{a} \check{y}_{\bar{x}}, y_{\bar{t}}\right)-\varphi\left(x, \check{t}, \check{u}, \check{a}(+1) \check{u}_{x}+\check{a} \check{u}_{\bar{x}}, u_{\bar{t}}\right) .
\end{aligned}
$$

Taking account of the differentiability of the function $f(x, t, u, p, q)$ with respect to the arguments $u, p$ and $q$, and using the mean value theorem, we obtain

$$
B_{1}=\frac{\overline{\partial \varphi}}{\partial \bar{u}} z+\frac{\overline{\partial \varphi}}{\partial p}\left(a^{(+1)} z_{x}+a z_{\bar{x}}\right)+\frac{\overline{\partial \varphi}}{\partial q} z_{\bar{t}},
$$

where the line on top indicates that the value of the derivative is taken for certain 
mean values of the arguments $u, p$ and $q$. Because of the linearity of the functional $F[\mu]$ we have

$$
\begin{gathered}
\frac{\partial \varphi}{\partial u}=F\left[\frac{\partial f}{\partial u}(x+s h, t, u, p, q)\right], \quad \frac{\partial \varphi}{\partial p}=F\left[\frac{\partial f}{\partial p}(x+s h, t, u, p, q)\right], \\
\frac{\partial \varphi}{\partial q}=F\left[\frac{\partial f}{\partial q}\right] .
\end{gathered}
$$

Using similar reasoning for the difference $B_{2}$ we obtain from the identity (32) the following linear difference equation for the net function $(z(x, t)$ :

where

$$
\overline{\mathscr{P}}_{h \mathrm{r}}^{\alpha} z=\left(a z_{\bar{x}}\right)_{x}^{(\alpha)}+Q(z)-\varrho z_{\bar{t}}^{-}=-\Psi \text { on } \Omega,
$$

$$
\begin{gathered}
Q(z)=b_{11} z_{x}+b_{12} \check{z}_{x}+b_{22} z_{\bar{x}}+b_{21} \check{z}_{\bar{x}}+d_{1} z+d_{2} \check{z} \\
b_{11}=\alpha a^{(+1)} \frac{\overline{\partial \varphi}}{\partial p}, \quad b_{12}=(1-\alpha) \check{a}(+1) \frac{\overline{\partial \varphi^{*}}}{\partial p}, \quad b_{22}=\alpha a \frac{\overline{\partial \varphi}}{\partial p}, \\
b_{21}=(1-\alpha) \check{a} \frac{\overline{\partial \varphi^{*}}}{\partial p}, \\
d_{1}=\alpha \frac{\overline{\partial p}}{\partial u}, \quad d_{2}=(1-\alpha) \frac{\overline{\partial \varphi^{*}}}{\partial u}, \quad \varphi^{*}=\varphi(x, \check{t}, \check{u}, \check{p}, q), \\
\varrho=-\alpha \frac{\overline{\partial \varphi}}{\partial q}-(1-\alpha) \frac{\partial \varphi^{*}}{\partial q} .
\end{gathered}
$$

The net function $\Psi=\Psi(x, t)$ is obviously the approximation error in the class of solutions $u=u(x, t)$ of the differential equation (24) and can be written in the form:

$$
\begin{aligned}
& \Psi=\psi^{(\alpha)}+\psi_{q}, \quad \psi=\psi_{a}+\psi_{p}+\psi_{\varphi}, \quad \psi_{q}=\alpha \psi_{q}^{*}+(1-\alpha) \psi_{q}^{* *}, \\
& \psi_{a}=\left(a u_{\bar{x}}\right)_{x}-\left(k u^{\prime}\right)^{\prime} \text {, } \\
& \psi_{p}=\varphi\left(x, t, u, a^{(+1)} u_{x}+a u_{\tilde{x}}, \dot{u}\right)-\varphi\left(x, t, u, 2 k u^{\prime}, \dot{u}\right), \\
& \psi_{\varphi}=\varphi\left(x, t, u, 2 k u^{\prime}, \dot{u}\right)-f\left(x, t, u, 2 k u^{\prime}, \dot{u}\right) \\
& \psi_{q}^{*}=\varphi\left(x, t, u, a^{(+1)} u_{x}+a u_{\bar{x}}, u_{\bar{t}}\right)-\varphi\left(x, t, u, a^{(+1)} u_{x}+a u_{\bar{x}}, \dot{u}\right), \\
& \psi_{q}^{* *}=\varphi\left(x, \check{t}, \check{u}, \check{a}^{(+1)} \breve{u}_{x}+\check{a}^{2} \check{u}_{\bar{x}}, u_{\bar{t}}\right)-\varphi\left(x, \check{t}, \check{u}, \check{a}^{(+1)} \check{u}_{x}+\check{a} \check{u}_{\bar{x}}, \check{\breve{u}}\right) .
\end{aligned}
$$

\section{Approximation error of initial schemes}

Let us investigate the asymptotic behaviour of the approximation error $\Psi$ for any scheme $\mathscr{P}_{h \tau}^{\alpha}$ of an initial family when $h \rightarrow 0$ and $\tau \rightarrow 0$. The asymptotic order of $\psi$ depends on the properties of the pattern functionals of this scheme, and the order of $\psi_{q}$ on the values of the parameter $\alpha$ (of course, when the solution $u(x, t)$ of the differential equation (1) and the functions $k(x, t)$ and $f(x, t, u, p, q)$ are differentiable the required number is 1 ).

LEMMA 1. If in a fixed neighbourhood of the point $(x, t)$ of the net $\Omega$ the functions

$$
k^{\prime \prime}(x, t), \quad f^{\prime}(x, t, u, p, q), \quad u^{\prime \prime \prime}(x, t)
$$


satisfy Lipshitz's condition for $x$, and the function $\dot{u}(x, t)$ satisfies the condition for $t$, then for sufficiently small $h$ and $\tau$

$$
\Psi=O\left(h^{2}\right)+O(\tau) \text { for } 0 \leqslant \alpha \leqslant 1 .
$$

(The dash indicates partial derivatives with respect to $x, \dot{u}=\partial u / \partial t$ ).

It will be remembered first of all that, by hypothesis, the function $f(x, t, u, p, q)$ has derivatives $\partial f / \partial u, \partial f / \partial p, \partial f / \partial q$ that are finite for any values of $u, p$ and $q$.

For any initial scheme we have

$$
\begin{gathered}
a=k-0 \cdot 5 h k^{\prime}+h^{2}\left(\frac{k^{\prime \prime}}{2} A_{1}\left[s^{2}\right]+\frac{\left(k^{\prime}\right)^{2}}{k} A_{2}[s]\right)+Q\left(h^{3}\right), \quad a(+1)=a+h k^{\prime}+O\left(h^{\mathrm{a}}\right), \\
\varphi(x, t, u, p, q)=f(x, t, u, p, q)+h f^{\prime}(x, t, u, p, q) F[s]+O\left(h^{2}\right)=f(x, t, u, p, q)+O\left(h^{2}\right),
\end{gathered}
$$

i.e. $\psi_{\varphi}=O\left(h^{2}\right)$. With the expansion

we find

$$
u_{x}=u^{\prime}+0 \cdot 5 h u^{\prime \prime}+\frac{h^{2}}{6} u^{\prime \prime \prime}+O\left(h^{3}\right), \quad u_{x}=u^{\prime}-0 \cdot 5 h u^{\prime \prime}+\frac{h^{2}}{6} u^{\prime \prime \prime}+O\left(h^{3}\right),
$$

and hence

$$
\psi_{a}=O\left(h^{2}\right), \quad a^{(+1)} u_{x}=a u_{\bar{x}}-2 k u^{\prime} O\left(h^{2}\right)
$$

$$
\psi_{p}=\frac{\overline{\partial \varphi}}{\partial p}\left(a(+1) u_{x}+a u_{\bar{x}}-2 k u^{\prime}\right)=O\left(h^{2}\right)
$$

where the bar above means that the derivative is taken for a mean value of the argument $p$. Thus, for any values of the parameter $\alpha$

$$
\psi=\psi_{a}+\psi_{p}+\psi_{\varphi}=O\left(h^{2}\right) .
$$

Using the mean value theorem we obtain

$$
\psi_{q}=\alpha \frac{\overline{\partial \varphi}}{\partial q}\left(u_{i}-\dot{u}\right)+(1-\alpha) \frac{\overline{\partial \varphi^{*}}}{\partial q}\left(u_{\tau}-\check{\ddot{u}}\right)=O(\tau),
$$

Since $u_{i}=\dot{u}+O(\tau)=\check{u}+O(\tau)$. Q.E.D.

LEMMA 2. If the conditions of Lemma 1 are satisfied and, in addition, the functions $k, u^{\prime}, \ddot{u}$. satisfy Lipshitz's condition for $t$, and the derivative $\partial f / \partial q$ satisfies the condition for $t, u, p$ and $q$, then

where

$$
\Psi=O\left(h^{2}\right)+O\left(\tau^{m_{\alpha}}\right)+O(h \tau), \quad \text { i. e. }|\Psi| \leqslant M\left(h^{2}+\tau^{m_{\alpha}}\right),
$$

$$
m_{\alpha}=\left\{\begin{array}{l}
2, \alpha=0.5 \\
1, \alpha \neq 0.5
\end{array}\right.
$$

To prove the lemma it is sufficient to prove that

$$
\psi_{q}=O\left(\tau^{m}\right)+O(h \tau)
$$

From the conditions of the lemma we can write

$$
\begin{aligned}
& \varphi\left(x, t, u, a^{(+1)} u_{x}+a u_{\bar{x}}, u_{\bar{t}}\right)-\varphi\left(x, t, u, a^{(+1)} u_{x}+a u_{\bar{x}}, \dot{u}\right) \\
& =\frac{\partial \varphi}{\partial q}\left(x, t, u, a(+1) u_{x}+a u_{\bar{x}}, \dot{u}\right)\left(u_{t}-\ddot{u}\right)+O\left(\tau^{2}\right), \\
& \frac{\partial \varphi}{\partial q}\left(x, \check{t}, \check{u}, \check{a}^{(+1) \check{u}_{x}}+\check{a} \check{u}_{\check{x}}, \check{u}\right)=\frac{\partial \varphi}{\partial q}\left(x, t, u, a^{(+1)} u_{x}+a u_{\breve{x}}, \ddot{u}\right)+O(\tau)+O(h),
\end{aligned}
$$


taking into account that

$$
a u_{\bar{x}}-\check{a} \check{u}_{\bar{x}}=k u^{\prime}-\check{k} \breve{u}^{\prime}+O(h)=O(\tau)+O(h)
$$

For $\psi_{q}$ we obtain the expression

$$
\psi_{q}=\alpha \psi_{q}^{*}+(1-\alpha) \psi_{q}^{* *}=\frac{\partial m}{\partial q}\left(x, t, u, a^{(+1)} u_{x}+a u_{\bar{x}}, \ddot{u}\right)(\alpha-0.5) \ddot{u} \tau+O\left(t^{2}\right)+O\left(h_{i}\right) .
$$

whence it can be seen that only when $\alpha=0.5$

$$
\psi_{q}=O\left(\tau^{2}\right) O\left(h_{\tau}\right)
$$

Since $h \tau<h^{2}+\tau^{2}$ we obtain $|\Psi| \leqslant M\left(\mathrm{~h}^{2}+\tau^{2}\right)$.

As can be seen from (43) Lemma 2 is useful only for symmetrical (with respect to $t$ ) schemes when $\alpha=0.5$, because when $\alpha \neq 0.5$ the order of approximation of this scheme cannot be increased by increasing the smoothness of the solution $u(x, t)$ and the functions $k(x, t)$ and $f(x, t, u, p, q)$. For this reason we shall always assume that the conditions of Lemma 2 are satisfied only for the schemes $P_{h z}^{0.5}(\alpha=0.5)$, and the conditions of Lemma 1 for all schemes $P_{h z}^{\alpha}$ when $0 \leqslant \alpha \leqslant 1$. We shall also assume that the conditions of Lemmas 1 and 2 are satisfied in each of the domains $\Delta_{v}\left(v=0,1, \ldots \ldots, v_{0}\right)$ into which the principal domain $\bar{д}$ is divided by the lines of discontinuity $\Gamma_{y}$ of the coefficient $k(x, t)$ (for more details on this see $\S 3.2$ ).

Lemmas 1 and 2 are also true for scheme $\left(25_{1}\right)$, and Lemma 1 is true for scheme $\left(25_{2}\right)$.

\section{\$2. DIFFERENCE BOUNDARY PROBLEM}

\section{Difference boundary problems of the third kind}

In order to formulate a difference problem corresponding to the boundary problem (I) for the differential equation $\mathcal{P} u=0$, it is necessary to write the difference boundary conditions when $x=0$ and $x=1$. The conditions of the first kind $u(0, t$ $=u_{1}(t), u(1, t)=u_{2}(t)$, considered in [3], can be represented exactly on the difference net, if we put $y(0, t)-y_{0}-u_{1}(t)$ and $y(1, t)=y_{N}=u_{2}(t)$; for the function $z$ $=y-u$, where $y$ is the solution of the difference problem and $u$ the solution of the problem (I), we obtain the homogeneous boundary conditions $z_{0}=0, z_{N}=0$. Hence the error in $z$ for the solution of the first difference boundary problem depends only on the approximation error $\Psi$ of the scheme $\mathcal{P}_{h \tau}^{a}$. We cannot accurately satisfy boundary conditions of the third kind on the difference net. The function $z$ will therefore also depend on the error due to approximation of the boundary conditions by the difference conditions. The difference boundary conditions must naturally have the same order of approximation as the scheme itself. Incidentally, direct substitution of the derivatives occurring in conditions of the third kind leads to difference boundary conditions of the first order of the approximation only. We shall illustrate this with an example of a boundary problem of the third kind for an ordinary differential equation

$$
\begin{aligned}
\left(k(x) u^{\prime}\right)^{\prime} & =-f(x), & 0<x & <1, \\
k u^{\prime}-\sigma_{1} u & =u_{1}, & x & =0, \\
k u^{\prime}+\sigma_{2} u & =u_{2}, & x & =1 .
\end{aligned}
$$


Let us examine the case of the boundary condition for $x=0$. We shall consider the difference boundary condition

$$
a_{1} y_{x, 0}-\sigma_{1} y_{0}=u_{1}
$$

and calculate the approximation error

$$
y=\left(a_{1} u_{x, 0}-\sigma_{1} u_{0}\right)-\left(k_{0} u_{0}^{\prime}-\sigma_{1} u_{0}\right)=a_{1} u_{x, 0}-k_{0} u_{0}^{\prime} \quad\left(k_{0}=k(0) \text { etc }\right) .
$$

Taking into account that $a_{1}=k_{0}+0 \cdot 5 h k_{0}^{\prime}+O\left(h^{2}\right), \quad u_{x, 0}-u_{0}^{\prime}+0 \cdot 5 h u_{0}^{\prime \prime}+O\left(h^{2}\right)$ we find that

$$
\boldsymbol{y}=0.5 h\left(k u^{\prime}\right)_{0}^{\prime}+O\left(h^{2}\right)=O(h) .
$$

Using this expression it is easy to write a difference boundary condition with a second order of approximation in the class of solutions $u=u(x)$ of the differential equation (1). From (1) we write

$$
v=-0 \cdot 5 h f_{0}+O\left(h^{2}\right)
$$

and consider the difference operator

$$
a_{1} y_{x, 0}-\sigma_{1} y_{0}+0 \cdot 5 h f_{0}=u_{1} .
$$

It follows from the above reasoning that this operator has a second order of approximation in the class of solutions of a differential equation satisfying condition (2).

Now let us examine the third boundary problem for (1.1):

$$
\begin{aligned}
& \mathscr{P} u=\left(k u^{\prime}\right)^{\prime}+f\left(x, t, u, 2 k u^{\prime}, \dot{u}\right)=0, \\
& l^{(1)} u=k u^{\prime}-\sigma_{1}(t) u=u_{1}(t), \quad x=0, \\
& l^{(2)} u=k u^{\prime}+\sigma_{2}(t) u=u_{2}(t), \quad x=1
\end{aligned}
$$

(here $\left.u^{\prime}=\frac{\partial u}{\partial x}, \dot{u}=\frac{\partial u}{\partial t}\right)$.

The difference boundary conditions satisfying (6) and (7) can be written directly by analogy with (4).

$$
\begin{aligned}
& l_{h}^{(1)} y=\left(a^{(+1)} y_{x}-\sigma_{1} y\right)^{(\alpha)}+ 0 \cdot 5 h\left[\alpha f\left(0, t, y, 2 k y_{x}, y_{\bar{t}}\right)+\right. \\
&\left.+(1-\alpha) f\left(0, \check{t}, \check{y}, 2 \check{k} \check{y}_{x}, y_{\bar{t}}\right)\right]=u_{1}^{(\alpha)}(t) \text { for } x=0, \\
& l_{h}^{(2)} y=\left(a y_{\bar{x}}+\sigma_{2} y\right)^{(\alpha)}-0.5 h\left[\alpha f\left(1, t, y, 2 k y_{\breve{x}}, y_{\bar{t}}\right)+\right. \\
&\left.+(1-\alpha) f\left(1, \check{t}, \check{y}, 2 \check{k} \check{y}_{\bar{x}}, y_{\bar{t}}\right)\right]=u_{2}^{(\alpha)}(t) \text { for } x=1,
\end{aligned}
$$

where $t=t-\tau$ (the arguments of the functions $k, y, y_{x}, y_{\bar{x}}$ will not be indicated). We may point out that $2 a^{(+1)} y_{x}$ (or $2 a y_{\bar{x}}$ ) can be written instead of $2 k y_{x}$ (or $2 k y_{\bar{x}}$ ) in (8) and (9) (see section 4).

We give below some other boundary conditions equivalent to (8) in its order of approximation:

$$
\begin{gathered}
l_{h}^{(1)} y=\left(a^{(+1)} y_{x}-\sigma_{1} y\right)^{(\alpha)}+0 \cdot 5 h f\left(0, t^{(\alpha)}, y^{(\alpha)}, 2\left(k, y_{x}\right)^{(\alpha)}, y_{\bar{t}}\right)=u_{1}^{(\alpha)}, \\
l_{h}^{(1)} y=\left(a^{(+1)} y_{x}-\sigma_{1} y\right)^{(\alpha)}+0 \cdot 5 h f\left(0, \check{t}, 2 \check{k}_{\bar{y}}, y_{\bar{t}}\right)=u_{1}^{(\alpha)} .
\end{gathered}
$$


From the boundary condition (6) we have

$$
f\left(0, t, u, 2 k u^{\prime}, \dot{u}\right)=f\left(0, t, u, 2 \sigma_{1} u+2 u_{1}, \dot{u}\right) .
$$

Instead of $2 k y_{x}$ (or $2 a^{(+1)} y_{x}$ ) in (8) we can write $2 \sigma_{1} y+2 u_{1}$. The boundary condition for $x=0$ will then have the form

$$
\begin{aligned}
l_{h}^{(1)} y=\left(a^{(+1)} y_{x}-\sigma_{1} y\right)^{(\alpha)}+0 \cdot 5 h[\alpha & f\left(0, t, y, 2 \sigma_{1} y+2 u_{1}, y_{\bar{t}}\right)+ \\
+ & \left.(1-\alpha) f\left(0, \check{t}, \check{y}, 2 \check{\sigma}_{1} \breve{y}+2 \check{u}_{1}, y_{\grave{t}}\right)\right]=u_{i}^{(\alpha)},
\end{aligned}
$$

or

$$
l_{h}^{(1)} y=\left(a^{(+1)} y_{x}-\sigma_{1} y\right)^{(\alpha)}+0 \cdot 5 h f\left(0, t, \check{y}, 2 \check{\sigma}_{1} \check{y}+2 \check{u}_{1}, y_{\bar{t}}\right)=u_{1}^{(\alpha)} .
$$

As already mentioned boundary conditions $(8),\left(8_{1}\right)-\left(8_{4}\right)$ have the same order of approximation.

We prefer to take the boundary condition in the form (8), although $\left(8_{2}\right),\left(8_{3}\right)$ and $\left(8_{4}\right)$ are simpler, because it is easier to pass on to the case of a non-linear boundary condition of the form

$$
k u^{\prime}=\chi(t, u, \dot{u}) .
$$

All the results obtained for condition (8) can be easily adopted to any of the boundary conditions $\left(8_{1}\right)-\left(8_{4}\right)$.

We write the approximation error of the boundary condition

$$
v_{1}=l_{k}^{(1)} u-\left(l^{(1)} u\right)^{(\alpha)}
$$

in the form of a sum

where

$$
v_{1}=\left(v_{a}+v_{p}\right)^{(a)}+v_{q},
$$

$$
\begin{aligned}
v_{a} & =a^{(+1)} u_{x}-k u^{\prime}+0.5 h f\left(0, t, u, 2 k u_{x}, \dot{u}\right), \\
v_{p} & =0.5 h\left[f\left(0, t, u, 2 k u_{x}, \dot{u}\right)-f(0, t, u, 2 k u, \dot{u})\right], \\
v_{q} & =\alpha v_{q}^{*}+(1-\alpha) v_{q}^{* *}, \\
v_{q}^{*} & =0 \cdot 5 h\left[f\left(0, t, u, 2 k u_{x}, u_{\bar{t}}\right)-f\left(0, t, u, 2 k u_{x}, \dot{u}\right)\right], \\
v_{q}^{* *} & =0.5 h\left[f\left(0, \check{t}, \check{u}, 2 \check{k} \check{u}_{x}, u_{\bar{t}}\right)-f\left(0, \check{t}, \check{k} \check{u}_{x}, \check{u}\right)\right] .
\end{aligned}
$$

We shall find the condition for $z=y-u$, where $u$ is the solution of the differential equation (5) satisfying the boundary condition $l^{(1)} u=u_{1}$ and $y$ is the net function satisfying the condition $l_{h}^{(1)} y=u_{1}^{(\alpha)}$. For this purpose we use the identity

$$
l_{h}^{(1)} y-l_{h}^{(1)} u=-v_{1} .
$$

Using the mean value theorem we shall have

$$
\begin{gathered}
f\left(0, t, y, 2 k y_{x}, y_{\bar{t}}\right)-f\left(0, t, u, 2 k u_{x}, u_{\bar{t}}\right)=\frac{\overline{\partial f}}{\partial u} z+\frac{\overline{\partial f}}{\partial p} 2 k z_{x}+\frac{\partial \bar{f}}{\partial q} z_{\bar{t}}, \\
f\left(0, \check{t}, \check{y}, 2 \check{k}_{y_{x}}, y_{\grave{t}}\right)-f\left(0, \check{t}, \check{u}, 2 \check{k}_{u_{x}}, u_{\bar{\imath}}\right)=\frac{\overline{\partial f^{*}}}{\partial u} \check{z}+\frac{\overline{\partial f^{*}}}{\partial p} 2 \check{k}_{\bar{k}}+\frac{\overline{\partial f^{*}}}{\partial q} z_{\bar{t}},
\end{gathered}
$$


where

$$
\begin{array}{cl}
\quad \frac{\partial f}{\partial u}=\frac{\partial f}{\partial u}(0, t, \bar{u}, \bar{p}, \bar{q}), \quad \frac{\overline{\partial f}}{\partial u}=\frac{\partial f}{\partial u}\left(0, \check{t}, \overline{u^{*}}, \overline{p^{*}}, \overline{q^{*}}\right) \text { etc. } \\
\bar{u}=u+\theta_{1} z, \quad \bar{p}=2 k\left(u_{x}+\theta_{1} z_{x}\right), \quad \bar{q}=u_{\bar{t}}+\theta_{1} z_{\bar{t}}, \quad 0 \leqslant \theta_{1} \leqslant 1, \\
\bar{u}^{*}=\check{u}+\theta_{2} \check{z}, \quad \bar{p}^{*}=2 \check{k}\left(\check{u}_{x}+\theta_{2} \check{z}_{x}\right), \quad \bar{q}^{*}=u_{\bar{t}}+\theta_{2} z_{\bar{t}}, \quad 0 \leqslant \theta_{1} \leqslant 1 .
\end{array}
$$

Substituting (17) and (18) in (16) we shall have for $z$ the linear boundary condition

where

$$
l_{1} z=\left(a^{(+1)} z_{x}-\sigma_{1} z\right)^{(x)}+h q_{1}(z)-\varepsilon_{1} z_{\bar{t}}=-v_{1} \text { for } x=0,
$$

$$
\left.\begin{array}{c}
q_{1}(z)=\zeta_{11} z+\zeta_{12} \check{z}+\lambda_{11} z_{x}+\lambda_{12} \check{z}_{x}, \\
\zeta_{11}=0.5 \alpha \frac{\partial \bar{f}}{\partial u}, \quad \zeta_{12}=0.5(1-\alpha) \frac{\overline{\partial f^{*}}}{\partial u}, \quad \lambda_{11}=\alpha k \frac{\overline{\partial f}}{\partial p}, \\
\lambda_{12}=(1-\alpha) \check{k} \frac{\overline{\partial f^{*}}}{\partial p}, \quad \mathcal{E}_{1}=-0.5 h\left(\alpha \frac{\overline{\partial f}}{\partial q}+(1-\alpha) \frac{\overline{\partial f^{*}}}{\partial q}\right) .
\end{array}\right\}
$$

Taking into account that

$$
\frac{\partial f}{\partial q} \leqslant-m \text { or }-\frac{\partial f}{\partial q} \geqslant m>0,
$$

we find

$$
\varepsilon_{1} \geqslant m h>0,
$$

where $m$ is a positive constant independent of $h$. Since the derivatives $\partial f / \partial u$ and $\partial f / \partial p$ are finite,

$$
\left|\zeta_{s k}\right| \leqslant M, \quad\left|\lambda_{s k}\right| \leqslant M, \quad s, k=1,2 .
$$

For $x=1$ at the other end there is the similar condition

$$
\begin{gathered}
l_{2} z=\left(a z_{\bar{x}}+\sigma_{2} z\right)^{(\alpha)}-h q_{2}(z)+\varepsilon_{2} z_{\bar{t}}=-\nu_{2} \text { for } x=1, \\
q_{2}(z)=\zeta_{22} z+\zeta_{21} \check{z}+\lambda_{22} z_{\bar{x}}+\lambda_{21} \check{\check{z}}_{\bar{x}} .
\end{gathered}
$$

The approximation error $y_{2}$ is determined from formulae similar to (10)-(15).

In investigating the order of accuracy of a difference problem corresponding to problem (I) we shall require the asymptotic expansion of $v_{1}$ and $\nu_{2}$ when $h \rightarrow 0$ and $\tau \rightarrow 0$.

LEMMA 3. If the following conditions are satisfied:

1. The function $\frac{\partial f}{\partial p}(0, t, u, p, q)$ satisfies Lipshitz's condition for $p$, and $\frac{\partial f}{\partial q}$ $(0, t, u, p, q)$ the condition for $q$

2. The functions $k^{\prime \prime}, u^{\prime \prime \prime}$ satisfy Lipshitz's condition for $x$ in the fixed neighbourhood of the boundary $x=0(0 \leqslant x<\bar{x}, 0 \leqslant t \leqslant T)$

3. $\dot{u}(0, t)$ satisfies Lipshitz's condition for $t$, then the approximation error of the boundary condition (8) in the class of solutions $u(x, t)$ of the differential equation (5) satisfying the condition (6) has the form

$$
v_{1}=\bar{v}_{1}^{(\alpha)}+O(h \tau)+O\left(h^{3}\right) .
$$


where

$$
\begin{aligned}
& \bar{v}_{1}=h^{2}\left\{\frac{k u^{\prime \prime \prime}}{6}+\frac{k^{\prime} u^{\prime \prime}}{4}+\left(\frac{k^{\prime \prime}}{2} A_{1}\left[s^{2}\right]+\frac{\left(k^{\prime}\right)^{2}}{k} A_{2}[s]\right) u^{\prime}+\right. \\
& \left.-\begin{array}{ll}
1 & \partial f \\
4 & \partial p
\end{array}\left(0, t, u, 2 k u^{\prime}, \dot{u}\right) k u^{\prime \prime}\right\}_{x=0} ;
\end{aligned}
$$

and if', moreover, $\frac{\partial f}{\partial q}(0, t, u, p, q)$ satisfies Lipshitz's condition for $t, u, p$, and $\ddot{u}(0, t)$ the condition for $t$, then

where

$$
\nu_{1}=\bar{\nu}_{1}^{(\alpha)}+O\left(h \tau^{m_{\alpha}}\right)+O\left(h^{3}\right)
$$

$$
m_{z}= \begin{cases}2, & \alpha=0.5 \\ 1, & \alpha \neq 0.5\end{cases}
$$

Similar expressions can be obtained for $\nu_{2}$.

The error $v_{1}$ is determined from formulac (10)-(15). We shall show that $v_{a}+v_{p}=\bar{v}_{1}+O\left(h^{3}\right)$. From the expansions

$$
\begin{gathered}
a^{(+1)}=k+0 \cdot 5 h k^{\prime}+h^{2}\left(\frac{k^{\prime \prime}}{2} A_{1}\left[s^{2}\right]+\frac{\left(k^{\prime}\right)^{2}}{k} A_{\Omega}[s]\right)+O\left(h^{3}\right), \\
u_{x}=u^{\prime}+0 \cdot 5 h u^{\prime \prime}+\frac{h^{2}}{6} u^{\prime \prime \prime}+O\left(h^{3}\right),
\end{gathered}
$$

we find

From the relations

$$
v_{a}=\left[\frac{k u^{\prime \prime}}{6}+\frac{k^{\prime} u^{\prime \prime}}{4}+\left(\frac{k^{\prime \prime}}{2} A_{1}\left[s^{2}\right]+\frac{\left(k^{\prime}\right)^{2}}{k} A_{2}[s]\right) u^{\prime}\right] h^{2}+O\left(h^{3}\right) .
$$

$$
u_{x}-u^{\prime}=O(h), \quad a^{(+1)} u_{x}-k u^{\prime}=0 \cdot 5 h\left(k u^{\prime}\right)^{\prime}+O\left(h^{2}\right),
$$

$$
v_{p}=0 \cdot 5 h\left[f\left(0, t, u, 2 k u_{x}, \dot{u}\right)-f\left(0, t, u, 2 k u^{\prime}, \dot{u}\right)\right]
$$

it follows that

$$
=\frac{\partial f}{\partial p}\left(0, t, u, 2 k u^{\prime}, \dot{u}\right)\left(k u_{x}-k u^{\prime}\right) \cdot h+O\left(h^{3}\right)
$$

$$
v_{p}=\left.\frac{\partial f}{\partial p}\left(0, t, u, 2 k u^{\prime}, \dot{u}\right) k u^{\prime \prime}\right|_{x=0} h^{2}+O\left(h^{3}\right) .
$$

Combining this with (25) we obtain $v_{a}+v_{p}=\bar{v}_{1}+O\left(h^{3}\right) ; v_{q}$ can also be evaluated without difficulty. NoTE. If $\frac{\partial f}{\partial q}$ satisfies Hölder's condition of the order $\gamma \geqslant 0.5$ for $t, u, p$ and $q$, then

$$
v_{1}=\bar{y}_{1}^{(\alpha)}+O\left(h \tau^{1+\gamma}\right)+O\left(h^{3}\right) .
$$

This calculation is sufficient for the proof of Lemma 4 in $\S 3$.

\section{Statement of the difference boundary problem}

Having chosen the initial family of homogeneous difference schemes (see $\S 1.4$ ) and written the difference boundary conditions, we can formulate the difference boundary problem corresponding to (I) as follows:

Find a function $y=y(x, t)$ defined on the net $\bar{\Omega}$, satisfying the difference equation

$$
\begin{aligned}
\mathscr{P}_{h x}^{\alpha} y=\left(a y_{\bar{x}}\right)_{x}^{(\alpha)}+\alpha \varphi(x, t, & \left.y, a^{(+1)} y_{x}+a y_{\bar{x}}, y_{\bar{t}}\right)+ \\
& +(1-\alpha) \varphi\left(x, \check{t}, \check{y}, \check{a}^{(+1)} \check{y}_{x}+\check{a ̆}_{\bar{y}} y_{\bar{t}}\right)=0 \text { on } \Omega,
\end{aligned}
$$


the boundary conditions

and the initial condition

$$
\begin{array}{ll}
l_{h}^{(1)} y=u_{1}^{(\alpha)}(t), & x=0, \\
l_{h}^{(2)} y=u_{2}^{(\alpha)}(t), & x=1,
\end{array}
$$

$$
y(x, 0)=u_{0}(x)
$$

The continuity conditions (1.5) are absent in this formulation because we are considering a through computation scheme in which the lines of discontinuity of the coefficient $k(x, t)$ are not explicitly separated.

Because of conditions (1.6) and (1.7), and the normalization and monotonization conditions of pattern functionals (see $\S 1.5$ ), the following inequalities must be satisfied

$$
\begin{gathered}
M \geqslant a(x, t) \geqslant m>0, \quad 0<m \leqslant-\frac{\partial \varphi}{\partial q} \leqslant M, \quad\left|\frac{\partial \varphi}{\partial u}\right| \leqslant M, \quad\left|\frac{\partial \varphi}{\partial p}\right| \leqslant M, \\
\sigma_{1} \geqslant 0 \quad \sigma_{2} \geqslant 0, \quad \sigma_{2}+\sigma_{2} \geqslant m>0 .
\end{gathered}
$$

We shall call the difference problem defined by the conditions (26), (8), (9), (27)-(29), the problem (II).

\section{Difference boundary problem for the solution error}

To investigate the problem of the convergence and accuracy of the solution $y(x, t)$ of problem (II) as compared with the solution $u=u(x, t)$ of problem (I) it is necessary to evaluate the net function $z=y-u$ in terms of the approximation errors $\Psi, v_{1}$ and $v_{2}$. Taking into account the results obtained in $\S 1.6$ and $\S 1.2$, we see that $z(x, t)$ is a solution of the following difference boundary problems:

$$
\begin{aligned}
& \left(a z_{\bar{x}}\right)_{x}^{(\alpha)}+Q(z)-\varrho z_{\bar{f}}=-\Psi, \\
& l_{1} z=\left(a^{(+1)} z_{x}-\sigma_{1} z\right)^{(\alpha)}+h q_{1}(z)-\varepsilon_{1} z_{\bar{t}}=v_{1}, \quad x=0, \\
& l_{2} z=\left(a z_{\bar{x}}+\sigma_{2} z\right)^{(\alpha)}-h q_{2}(z)+\mathcal{C}_{2} z_{\bar{t}}=-\nu_{2}, \quad x=1 \text {, } \\
& z(x, 0)=0,
\end{aligned}
$$

where

$$
\left.\begin{array}{c}
Q(z)=b_{11} z_{x}+b_{12} \check{z}_{x}+b_{22} z_{\bar{x}}+b_{21} \check{z}_{\bar{x}}+d_{1} z+d_{2} \check{z}, \\
q_{1}(z)=\left.\left(\zeta_{11} z+\zeta_{12} \check{z}+\lambda_{11} z_{x}+\lambda_{12} \check{z}_{x}\right)\right|_{x=0 .} \\
q_{2}(z)=\left.\left(\zeta_{22} z+\zeta_{21} \check{z}+\lambda_{22} z_{\bar{x}}+\lambda_{21} \check{z}_{\bar{x}}\right)\right|_{x=1 .}
\end{array}\right)
$$

The coefficients $\varrho, a, b_{s k}, d_{s}(s, k=1,2)$, defined by formulae (1.35), and the coefficients $\varepsilon_{s}, \zeta_{s k}, \lambda_{s k}(s, k=1,2)$ defined by formulae (20)-(21) satisfy the conditions

$$
\begin{array}{cl}
0<m \leqslant 0 \leqslant M, & 0<m \leqslant a \leqslant M, \quad\left|b_{s k}\right| \leqslant M, \quad\left|d_{s}\right| \leqslant M, \\
\mathcal{E}_{s} \geqslant m h>0, \quad & \sigma_{s} \geqslant 0, \quad \sigma_{1}+\sigma_{2} \geqslant m>0, \quad\left|\zeta_{s k}\right| \leqslant M, \\
& \left|\lambda_{s k}\right| \leqslant M \quad(s, k=1,2) .
\end{array}
$$

The problem defined by (30)-(36) will be called problem (III). 
Here $\Psi$ is the approximation error of the scheme $\mathcal{P}_{h r}^{a}, v_{1}$ and $v_{2}$ are the approximation errors of the boundary conditions in the class of solutions of the differential equation (1.1). These functions are defined by (1.36)-(1.41) and (10)-(15).

\section{Solution of difference equations}

We introduce a computation scheme for the solution of the difference problem (II)

$$
\left.\begin{array}{c}
P_{h x}^{a} y=\left[\left(a y_{\bar{x}}\right)_{x}+b(x, t)\left(a(+1) y_{x}+a y_{\bar{x}}\right)-d(x, t) y+\varphi(x, t)^{(\alpha)}-(\varrho(x, t))^{(\alpha)} \cdot y_{\bar{t}}=0,\right. \\
l_{h}^{(1)} y=u_{1}^{(\alpha)} \text { for } x=0, \quad l_{h}^{(z)} y=u_{\mathrm{z}}^{(\alpha)} \text { for } x=1, \quad y(x, 0)=u_{0}(x) \text { for } t=0,
\end{array}\right\}
$$

corresponding to problem (I) for the linear equation (1.11). Assuming that $q(x, t) \geqslant 0$ and hence, $d(x, t) \geqslant 0$, we rewrite the difference equations and the boundary conditions (37) in the form

where

$$
\begin{gathered}
\left(a y_{\hat{x}}\right)_{x}-\left(d+\varrho^{(\alpha)} / \alpha \tau\right) y+b\left(a^{(+1)} y_{x}+a y_{\bar{x}}\right)=-F^{*}, \\
y_{0}=x_{1} y_{1}+\mu_{1}, \quad y_{N}=x_{2} y_{N-1}+\mu_{2},
\end{gathered}
$$

$$
\begin{aligned}
& F^{*}=\varrho^{(\alpha)} \check{y} / \alpha \tau+\frac{1-\alpha \alpha}{\alpha}\left[\left(\check{a} \check{y}_{\check{x}}\right)_{x}-\check{d} \check{y}+\check{\varphi}+\check{b}\left(\check{a}(+1) \check{y}_{x}+\check{a} \check{y}_{\check{x}}^{\check{x}}\right)\right]+\varphi(x, t), \\
& x_{1}=\bar{a}_{1} /\left(\vec{a}_{1}+\bar{\sigma}_{1} h+\varepsilon_{1} h / \alpha \tau\right), \quad x_{2}=\bar{a}_{N} /\left(\bar{a}_{N}+\vec{\sigma}_{9} h+\varepsilon_{9} h / \alpha \tau\right), \\
& \bar{a}_{1}=a_{1}\left(1+0.5 h b_{1}\right), \quad \bar{a}_{N}=a_{N}\left(1-0.5 h b_{N-1}\right), \quad \bar{\sigma}_{1}=\sigma_{1}+0.5 h q_{0}, \quad \bar{\sigma}_{1}=\sigma_{2}+0.5 h q_{N}, \\
& \mu_{1}=\varkappa_{1}\left\{h \mathcal{E}_{1} / \alpha \bar{a}_{1} \tau+\left(\check{a}_{1} \check{y}_{x, 0}+\check{\sigma}_{1} \check{y}_{0}\right)(1-\alpha) / \alpha \bar{a}_{1}+\left(u_{1}+0 \cdot 5 h f_{0}\right)^{(\alpha)} / \alpha\right\} \text {. } \\
& f_{0}=f(0, t), \quad q_{0}=q(0, t), \quad q_{N}=q(1, t), \quad y_{0}=y(0, t), \quad a_{1}=a(h, t) \text { etc. }
\end{aligned}
$$

We shall not write the expression for $\mu_{2}$.

Because of the conditions $\sigma_{1} \geqslant 0, \sigma_{2} \geqslant 0, \sigma_{1}+\sigma_{2} \geqslant m>0, \varepsilon_{1} \geqslant m h>0, \varepsilon_{2}>m h>0$, $q \geq 0$ one of two cases is possible (when $\bar{a}>0$, see (43)):

$$
\text { 1) } 0 \leqslant x_{1}<1, \quad 0 \leqslant x_{2} \leqslant 1 ; \quad \text { 2) } 0 \leqslant x_{1} \leqslant 1, \quad 0 \leqslant x_{2}<1 \text {. }
$$

The solution of the problem is known at the moment $t-\tau$. Hence the right-hand side of $F^{*}$ and the coefficients $\chi_{1}, \varkappa_{2}, \mu_{1}$ and $\mu_{2}$ arc known while determining the values of $y=y(x, t)$ on a new row of $t$. Consequently all the calculations on each row are reduced to a solution of the difference boundary problem

where

$$
\begin{gathered}
A_{i} y_{i-1}-C_{i} y_{i}+B_{i} y_{i+1}=-F_{i}, \quad 0<i<N, \\
y_{0}=x_{1} y_{1}+\mu_{1}, \quad y_{N}=x_{2} y_{N-1}+\mu_{2},
\end{gathered}
$$

$$
\begin{gathered}
F_{i}=h^{2} F_{i}^{*}, \quad A_{i}=a_{i}\left(1-0.5 h b_{i}\right), \quad B_{i}=a_{i+1}\left(1+0 \cdot 5 h b_{i}\right), \\
C_{i}=A_{i}+B_{i}+h^{2} D_{i}, \quad D_{i}=d_{i}+Q_{i}^{(\alpha)} / \alpha \tau, \\
a_{i}=a\left(x_{i}, t\right), \quad b_{i}=b\left(x_{i}, t\right) \text { etc. }
\end{gathered}
$$

The problem defined by (41)-(42) can be solved if

$$
A_{i}>0, \quad B_{i}>0, \quad D_{i} \geqslant 0 .
$$

The conditions $A_{i}>0, B_{i}>0$ will be satisfied if $1-0.5 h b_{i}>0$ or $h<h_{0}=\min 2 /\left|b_{i}\right|$, i.e. if the $x$-net is sufficiently fine. If $b_{l} \equiv 0$, then the condition $h<h_{0}$ disappears.

The difference boundary problem defined by (41)-(42) the coefficients of which satisfy the condition (43), and hence the conditions (40), can be solved either by one of the iterative methods, or the non-iterative method of successive substitution widely used in recent years in computation work (see [17]-[19], [2], [11]). We shall consider this method in greater detail. 
1. The dispersive formulae for the right-hand side are

$$
\begin{gathered}
\vec{\alpha}_{i+1}=\frac{B}{B_{i}+\left(1-\alpha_{i}\right) A_{i}+h^{2} D_{i}}, \quad 1<i<N, \quad \alpha_{1}=x_{1}, \\
\vec{\beta}_{i+1}=\frac{\alpha_{i+1}}{B_{i}}\left(A_{i} \beta_{i}+F_{i}\right), \quad 1<i<N, \quad \beta_{1}=\mu_{1}, \\
\overleftarrow{y_{i}}=\alpha_{i+1} y_{i+1}+\beta_{i+1}, \quad 0<i<N, \quad y_{N}=\left(\mu_{2}+x_{2} \beta_{N}\right) /\left(1-\varkappa_{2} \alpha_{N}\right) .
\end{gathered}
$$

The arrows $\rightarrow$ and $\leftarrow$ indicate the direction of computation: $\rightarrow$ from $i$ to $i+1, \leftarrow$ from $i+1$ to $i$.

Formulae (44)-(46) can be derived by using either the factorization method [17], or the exclusion method (see [18], [19]) described below. The solution of our problem will be investigated in the form (46), where $\alpha_{i}$ and $\beta_{i}$ are coefficients to be determined. We write $y_{i-1}=\alpha_{i} y_{i}+\beta_{i}$ in equation (41):

$$
\left(A_{i} \alpha_{i}-C_{i}\right) y_{i}+B_{i} y_{i+1}+A_{i} \beta_{i}+F_{i}=0 ;
$$

then, using (46) we eliminate $y_{i}$ :

$$
\left[\left(A_{i} \alpha_{i}-C_{i}\right) \alpha_{i+1}+B_{i}\right] y_{i+1}+\left[\left(A_{i} \alpha_{i}-C_{i}\right) \beta_{i+1}+\left(A_{i} \beta_{i}+F_{i}\right)\right]=0 .
$$

Formulae (44) and (45) are obtained from the condition that the expressions in square brackets are equal to zero. The initial conditions $\alpha_{1}=x_{1}, \beta_{1}=\mu_{1}$ follow from the conditions $y_{0}=x_{1} y_{1}+$ $+\mu_{1}=\alpha_{1} y_{1}+\beta_{1}$, the expression for $y_{N}$ (from the formula $y_{N-1}=\alpha_{N} y_{N}+\beta_{N}$ and the boundary condition $y_{N}=x_{2} y_{N-1}+\mu_{2}$ after eliminating $y_{N-1}$.

Computations according to the formulae (44)-(46) are stable if conditions (40) and (43) are satisfied. All $\alpha_{i} \leqslant 1$ and $\alpha_{N}<1$, if $x_{1}<1$, and $\alpha_{N}=1$, if $x_{1}=1, D_{i}=0$. Therefore the expression $1-\varkappa_{2} \alpha_{N}$ is always positive.

2. The dispersive formulae for the left-hand side are:

$$
\begin{gathered}
\overleftarrow{\xi}_{i}=\frac{A_{i}}{A_{i}+\left(1-\xi_{i+1}\right) B_{i}+h^{2} D_{i}}, \quad 1<i<N, \quad \xi_{N}=x_{2}, \\
\overleftarrow{\eta}_{i}=\frac{\xi_{i}}{A_{i}}\left(B_{i} \eta_{i+1}+F_{i}\right), \quad 1<i<N, \quad \eta_{N}=u_{2}, \\
\overleftarrow{y_{i+1}}=\xi_{i+1} y_{i}+\eta_{i+1}, \quad 0 \leqslant i<N, \quad y_{0}=\left(\mu_{1}+x_{1} \eta_{1}\right) /\left(1-x_{1} \xi_{1}\right) .
\end{gathered}
$$

3. Cross-dispersive method. Sometimes, especially in manual multiplication (or when the internal store of the computer is small) it is convenient to use the cross-dispersive method (shuttle method) in which the computation is carried out independently by formulae (44), (45) and (47), (48) up to a certain $i=n$. The continuity conditions determine $y_{n}$ and computation is continued on the right from the point $i=n$ from formula (49) and, independently, on the left from formula (46). Suppose the quantities $\alpha_{n+1}, \beta_{n+1}, \xi_{n+1}$ and $\eta_{n+1}$ have been found. Then we shall have

$$
y_{n}=\alpha_{n+1} y_{n+1}+\beta_{n+1}, \quad y_{n+1}=\xi_{n+1} y_{n}+\eta_{n+1} .
$$

Eliminating $y_{n+1}$ we find

$$
y_{n}=\frac{\beta_{n+1}+\alpha_{n+1} \eta_{n+1}}{1-\alpha_{n+1} \eta_{n+1}}
$$

Knowing $y_{n}$, all $y_{i}$ for $\mathrm{i}<n$ can be found from formula (46), and all $\mathrm{y}_{i}$ for $i>n$ from formula (49).

Various iterative methods are generally used for solving non-linear difference equations and the dispersive method is used to compute the iterations. We cannot deal with this question here (see, for example, [10], [11] etc.). Scheme coefficients independent of the required function on the preceding line are sometimes taken to avoid iterations, so that a linear equation may be obtained for $y$ on the new row. In this case of course a scheme of the first order of approximation with respect to $\tau$ is obtained. 


\section{§ 3. ACCURACY OF THROUGH COMPUTATION SCHEMES}

1. Evaluation of the solution of problem (III)

In $\S 2$ the problem of the order of accuracy of a solution of problem (II) was reduced to an estimation of the solution of problem (III) for $z=y-u$ by using the approximation errors $\Psi, \nu_{1}$ and $\nu_{2}$.

The solution of problem (III) was evaluated with a less general formulation in [4] and [5].

THEOREM 1. If the following conditions are satisfied

$$
\begin{gathered}
a \leqslant M, \quad\left|a_{\bar{i}}\right| \leqslant M, \quad \sigma_{s} \leqslant M, \quad\left(\sigma_{s}\right)_{i} \mid \leqslant M, \quad s=1,2, \\
0.5 \leqslant \alpha \leqslant 1,
\end{gathered}
$$

then for the solution of problem (III) with the initial conditions

$$
\left.z\right|_{t=0}=z(x, 0)
$$

the following inequality is true when $\tau<\tau_{0}$ is sufficiently small

$$
\begin{aligned}
\|z(x, t)\|_{0} \leqslant\left[\left.\sum_{t^{\prime}=\mathbf{z}}^{t^{\prime}=t} \tau||_{1} z_{\bar{t}}(x, t)\right|_{2} ^{\prime 2}\right]^{\frac{1}{2}}+\tilde{M}\left(\|\left. z(x, t)\right|_{i_{2}}+\left.|| z_{\bar{x}}(x, t)\right|_{2}\right) \leqslant \\
\quad \leqslant M\left\{!|z(x, 0)|_{\overline{2}}+\left\|z_{\bar{x}}(x, 0)\right\|_{2}+\left[\sum_{t^{\prime}=\tau}^{t^{\prime}=t} \overline{\tau|| \Psi\left(x, t^{\prime}\right) \|_{2}^{2}}\right]^{\frac{1}{2}}\right\},
\end{aligned}
$$

where $M$ and $\tilde{M}$ are pasitive constants independent of $h$ and $\tau$,

$$
\begin{aligned}
& \left.{ }_{1}|z(x, t)|\right|_{\overline{2}}=\left.|| z(x, t)\right|_{2}+\gamma_{1}|z(0, t)|+\gamma_{2}|z(1, t)| \text {, } \\
& \gamma_{s}=0 \text { for } \sigma_{s}=0, \quad \gamma_{s}=1 \text { for } \sigma_{s} \geqslant m>0 \text {, } \\
& \widetilde{\mid \Psi(x, t) \|_{2}}=|| \Psi(x, t) \|_{2}+\left|\nu_{1}^{*}(t)\right|+{ }_{1}^{\prime} v_{2}^{*}(t) \mid, \\
& v_{s}^{*}(t)=\frac{1}{\sqrt{h}} v_{s}(t) \text { for } \mathcal{E}_{s} \geqslant m \dot{h}>0, \\
& \nu_{s}^{*}(t)=\nu_{s}(t) \text { for } \varepsilon_{s} \geqslant m>0 .
\end{aligned}
$$

Expression (3) is true for the first boundary problem $(z(0, t)=z(1, t)=0)$ if we formally put $v_{s} \equiv 0, s=1,2$.

Theorem 1 was proved in [4], [5] for the case $\zeta_{s k}=: 0, \lambda_{s k}=0(s, k=1,2)$, i.e. for the boundary conditions

$$
\left.\begin{array}{ll}
l_{1}^{*} z=\left(a^{(+1)} z_{x}-\sigma_{1} z\right)^{(a)}-\mathcal{E}_{1} z_{\bar{t}}=\nu_{1}, & x=0, \\
l_{z}^{*} z=\left(a z_{\bar{x}}+\sigma_{8} z\right)^{(x)}+\mathcal{E}_{\mathbf{z}} z_{\bar{t}}=-v_{8}, & x=1,
\end{array}\right\}
$$

and also for the operators

$$
Q(z)=b_{1} z_{x}+b_{2} \check{z}_{x} *+d_{1} z+d_{2} \check{z}, \quad z_{x} *=0.5\left(z_{\bar{x}}+z_{x}\right)
$$

and

$$
Q(z)=b_{1} z_{\bar{x}}+b_{2} \check{z}_{\bar{x}}+d_{1} z+d_{2} \check{z}
$$

The method of deriving (3) proposed in [5] is perfectly suitable in our case. The modifications necessary because the operator $Q(z)$ is more complex are so insignificant that we shall not discuss 
them. On replacing conditions (6) by conditions (2.31) and (2.32) on the right-hand side of identity (2.11) of [5] we have the additional term

$$
\begin{aligned}
\vec{R}=\tau h\left(\zeta_{11} z_{0}+\zeta_{12} \check{z}_{0}\right. & \left.+\lambda_{11} z_{x, 0}+\lambda_{12} \check{z}_{x, 0}\right) z_{\bar{t}, 0}+\tau h\left(\zeta_{22} z_{N}+\right. \\
& +\zeta_{21} \check{z}_{N}+\lambda_{32} z_{1} x, N \\
& \left.+\lambda_{21} \check{z}_{\bar{x}, N}\right) z_{\bar{t}, N} \quad\left(z_{0}=z(0, t), \quad z_{N}=z(1, t) \text { etc. }\right) .
\end{aligned}
$$

With the arguments usual for the procedure described in [4] we obtain the expression

$$
|\bar{R}| \leqslant \frac{\tau}{8}\left(\mathcal{E}_{1} z_{\bar{t}, 0}^{2}+\mathcal{E}_{2} z_{\bar{t}, N}^{2}\right)+M \tau(I+\check{I} .
$$

The main integral inequality (2.17) from [5] and all the subsequent calculations remain unchanged.

Evaluation of the right-hand side in terms of the norm $\|\Psi\|_{2}$ is too rough for proving the convergence of the solution of problem (II) when the heat-conduction coefficient $k(x, t)$ is discontinuous. An improved estimate was obtained in [5] for solving problem (III) with the additional assumptions

$$
\begin{array}{ccc}
\Psi=\bar{\psi}^{(\alpha)}, & \nu_{1}=\bar{\nu}_{1}^{(\alpha)}, & \nu_{2}=\bar{\nu}_{2}^{(\alpha)}, \\
\varrho \leqslant M, & \varepsilon_{1} \leqslant M, & \varepsilon_{2} \leqslant M
\end{array}
$$

and the norms

$$
|| \bar{\psi}\left\|_{5}=\right\| \bar{\psi}\left\|_{4}+\left|\bar{\nu}_{1}\right|+\left|\bar{\nu}_{2}\right|, \quad\right\| \bar{\psi}\left\|_{5^{*}}=\left|\bar{\psi} \|_{4^{*}}+\right| \bar{\nu}_{1}|+| \nu_{2} \mid .\right.
$$

THEOREM 2. If the conditions (1), (2), (9) and (10) are satisfied, then the following a priori estimate is true for the solution of problem (III) when $\tau<\tau_{0}$ is sufficiently small

$$
\begin{aligned}
\|z(x, t)\|_{0} \leqslant & M\left\{\|\bar{\psi}(x, 0)\|_{5}+\|\bar{\psi}(x, t)\|_{5^{*}}+\right. \\
& \left.+\left[\sum_{t^{\prime}=\tau}^{t^{\prime}=t} \tau\left(\left\|\bar{\psi}\left(x, t^{\prime}\right)\right\|_{5^{*}}+\| \bar{\psi}_{\bar{t}}\left(x, t^{\prime}\right)_{5^{*}}\right)^{2}\right]^{\frac{1}{2}}\right\}+\bar{M}\left[\sum_{t^{\prime}=\tau}^{t^{\prime}=t} \tau\|\bar{\psi}(x, t)\|_{5}^{2}\right]^{\frac{1}{2}},
\end{aligned}
$$

where $\bar{M}=0$ when $b_{11}=b_{12}=b_{22}=b_{21}=0$.

This theorem corresponds to Theorem 6 of [5] with the qualifications made above regarding $Q(z)$ and the boundary conditions. To prove it the solution of problem (III) must be written in the form of the sum $z=v+w$, where $w$ is the solution of the "stationary" problem

$$
\left(a w_{\bar{x}}\right)_{x}=-\bar{\psi}, \quad a_{1} w_{x, 0}-\sigma_{1} w_{0}=-\bar{v}_{1}, \quad a_{N} w_{\bar{x}, N}+\sigma_{2} w_{N}=v_{\mathbf{2}} .
$$

We then obtain the following conditions for $v$

$$
\begin{gathered}
\left(a v_{\bar{x}}\right)_{x}^{(a)}+Q(v)-\varrho v_{\bar{t}}=-\Psi^{*}, \quad \Psi^{*}=Q(w)-\varrho\left(w_{\bar{t}}\right), \\
l_{1} v=v_{1}^{*}, \quad v_{1}^{*}=\mathcal{E}_{1} w_{\bar{t}, 0}-0.5 h\left(\zeta_{11} w_{0}+\zeta_{12} \check{w}_{0}+\lambda_{11} w_{x, 0}+\lambda_{12} \check{w}_{x, 0}\right), \\
l_{2} v=-v_{2}^{*}, \quad v_{2}^{*}=\mathcal{E}_{\mathrm{z}} w_{\bar{t}, N}-0.5 h\left(\zeta_{22} w_{N}+\zeta_{21} \check{w}_{N}+\lambda_{22} w_{\bar{x}, N}+\lambda_{81} w_{\bar{x}, N}\right) .
\end{gathered}
$$

To evaluate the function $v(x, t)$ we use Theorem 1. Taking account of the expression obtained in $\S 1$ of [5],

$$
\begin{gathered}
\|w\|_{0} \leqslant M\|\bar{\psi}\|_{3^{*}}, \quad\left\|w_{\bar{t}}\right\|_{0} \leqslant M\|\bar{\psi}\|_{5^{*}}, \quad\left|w_{x, 0}\right| \leqslant M\|\bar{\psi}\|_{5^{*}}, \\
\left|w_{\bar{x}, N}\right| \leqslant M\|\bar{\psi}\|_{s^{*}}, \quad\left\|w_{\bar{x}}\right\|_{2} \leqslant M\|\bar{\psi}\|_{5},
\end{gathered}
$$


we find

$$
\|Q(w)\|_{2}<M\left(\|\bar{\psi}\|_{s^{*}}+\left\|\bar{\psi}_{\bar{t}}\right\|_{s^{*}}\right)+\bar{M}\|\bar{\psi}\|_{s} .
$$

NoTE. It can be seen from (12) that, when $b_{s k}=0(s, k=1,2)$ and $\bar{\Psi}(x, 0)=0$, the inequality (12) contains only $\|\psi\|_{s^{*}}$ and $\left\|\bar{\psi}_{\bar{t}}\right\|_{s^{*}}$.

\section{Properties of the solution and coefficients of problem ( $I$ )}

To simplify the formulation of the following theorems we shall always assume that the solution of problem (I) and the functions in the differential equation $\mathscr{P} u=0$ and the boundary conditions have differential properties. (over the entire domain $\bar{д}$ or in each of the domains $\Delta_{v}=\left(\eta_{v}(t)<x<\eta_{v+1}(t), 0<t \leqslant T\right)$, $v$ $\left.=0,1,2, \ldots, v_{0}\right)$, which ensure the maximum order of approximation of the scheme and the difference boundary conditions. These conditions will be formulated below.

It is assumed everywhere that the function $f(x, t, u, p, q)$ has finite derivatives $\frac{\partial f}{\partial u}, \frac{\partial f}{\partial p}, \frac{\partial f}{\partial q}$ for all values of the arguments (see (1.6)).

The functions $k(x, t)$ and $f(x, t, u, p, q)$ have discontinuities of the first kind with respect to the variables $(x, t)$ on a finite number of curves $\Gamma_{v}\left(x=\eta_{v}(t)\right.$, $\left.v=1,2, \ldots, v_{0}\right)$, which divide the domain $\bar{\not}$ into subdomains $\Delta_{v}$. The following notations will be used

$$
\begin{gathered}
k_{\text {л, }}=k\left(\eta_{v}(t)-0, t\right), \quad k_{\text {п, }}=k\left(r_{\nu}(t)+0, t\right), \\
f_{\text {л, }}=f\left(\eta_{v}(t)-0, t, u\left(\eta_{\nu}(t), t\right), 2\left(k u^{\prime}\right)_{\text {л, }}, \dot{u}_{\text {л, }}\right) \text { etc. }
\end{gathered}
$$

If a function $v(x, t)$ is considered in the closed domain

$$
\bar{\Delta}_{v}=\left(\eta_{v}(t) \leqslant x \leqslant \eta_{v+1}(t), 0 \leqslant t \leqslant T\right),
$$

it is assumed that $v\left(\eta_{v}(t), t\right)=v_{\Pi, \eta}, v\left(\eta_{v+1}(t), t\right)=v_{\pi, v+1}$.

The curve $\Gamma_{v}$ intersects each of the straight lines $t=j \tau, j=0,1, \ldots$, at the point $x=\eta_{v}(t)=x_{n_{v}}+\theta_{v} h$, where $0 \leqslant \theta_{v} \leqslant 1, x_{n_{v}}=n_{v} h, n_{v}$ is an integer, $\left(x_{n_{v}}, t\right)$ is a point of the net $\Omega$. The numbers $n_{v}$ and $\theta_{\nu}$, generally speaking, are functions of $h$ and $t, n_{v}=n_{v}(h, t)$ and $\theta_{\nu}=\theta_{v}(h, t)$. If the line $\Gamma$, is parallel to the $t$-axis (fixed discontinuity), $n_{v}$ and $\theta_{v}$ will depend only on $h ; n_{v}=n_{v}(h), \theta_{v}=\theta_{v}(h)$.

In this paper only fixed discontinuities are considered, i.e.

$$
x=\eta_{v}=\text { const. }\left(v=1,2, \ldots, v_{0}\right) \text { for } 0 \leqslant t \leqslant T .
$$

We shall assume:

$\left(\mathrm{K}_{1}\right)$. The conditions of lemmas 1 and 2 are satisfied in each of the domains $\bar{\Delta}_{*}$. Then the approximation error of the scheme $\mathscr{P}_{h i}^{\alpha}$ in the class of solutions of the equation $\mathscr{P} u=0$

$$
\Psi=O\left(h^{2}\right)+O\left(\tau^{m_{\alpha}}\right), \text { where } m_{\alpha}= \begin{cases}1, & \alpha=0.5, \\ 2, & \alpha \neq 0.5 .\end{cases}
$$

at all points of the net $\Omega$, except the points

$$
\left(x_{n_{v}}, t\right) \text { and }\left(x_{n_{v+1}}, t\right), \quad v=1,2, \ldots, v_{0} ; \quad t=\tau, 2 \tau, \ldots, L \tau=T .
$$


The asymptotic order $\Psi$ when $h \rightarrow 0$ and $\tau \rightarrow 0$ at the points mentioned is examined in section 4.

$\left(\mathrm{K}_{2}\right)$. The conditions of Lemma 3 by virtue of which the approximation errors of the boundary conditions have the order

$$
v_{s}=\bar{v}_{s}^{(\alpha)} h^{2}+O\left(h \tau^{m_{\alpha}}\right)+O\left(h^{3}\right), \quad s=1,2,
$$

where $\bar{\nu}_{s}$ is determined from formula (2.23), are satisfied.

$\left(\mathrm{K}_{3}\right)$. Lipschitz's condition is satisfied by the functions $\sigma_{1}(t)$ and $\sigma_{2}(t)$ for $t$ when $0 \leqslant t \leqslant T$ and by $k(x, t)$, in each of the domains $\vec{\Delta}_{v}$.

$\left(\mathrm{K}_{4}\right)$. The limiting values of the functions

$$
k, k^{\prime}, k^{\prime \prime}, u^{\prime}, u^{\prime \prime}, u^{\prime \prime \prime}
$$

satisfy Lipshitz's condition for $t$ along each of the lines $\Gamma_{v}\left(v=0,1, \ldots, v_{0}, v_{0}+1\right)$ and the limiting value of the function $\partial^{2} u / \partial x \partial t$ only along the internal lines for $v=1,2, \ldots, v_{0}$.

For the smooth function $k(x, t)$ and $f^{\prime}(x, t, u, p, q)$ condition $\left(\mathrm{K}_{1}\right)$ is satisfied over the entire domain $\bar{A}$ and condition $\left(\mathrm{K}_{4}\right)$ only when $v=0 \quad(x=0)$ and $\nu=v_{0}+1(x=1)$.

It follows from these conditions that the following inequalities are true for the coefficients of problem (III)

$$
0<m h \leqslant \mathscr{E}_{s} \leqslant M h, \quad 0<m \leqslant \varrho \leqslant M, \quad 0<m \leqslant a \leqslant M, \quad 0 \leqslant \sigma_{s}(t) \leqslant M,
$$

$$
\begin{gathered}
\left|\zeta_{s k}\right| \leqslant M, \quad\left|\lambda_{s k}\right| \leqslant M, \quad s, k=1,2, \\
\left|a_{\bar{t}}\right| \leqslant M, \quad\left|\left(\sigma_{s}\right)_{\bar{t}}\right| \leqslant M,
\end{gathered}
$$

Theorems 1 and 2 can therefore be applied when $0.5 \leqslant \alpha \leqslant 1$.

3. Effect of the approximation error of boundary conditions on the accuracy of solution of the difference problem

The solution of problem (III) can be written as the sum of two partial solutions $z=z_{1}+z_{2}$, where $z_{1}$ is a solution of the problem with the homogeneous boundary condition $\left(v_{1} \equiv 0, v_{2} \equiv 0\right)$, and $z_{2}$ is a solution of problem (III) with a homogeous equation $(\Psi=0)$.

As mentioned in section 2 , we are considering fixed discontinuities only of the coefficient $k(x, t)$.

LEMMA 4. When $\tau<\tau_{0}$ is sufficiently small the following expression is true for the error $z_{2}$ of the solution of 'problem (II) because of the approximation error of' boundary conditions

$$
\left\|z_{2}(x, t)\right\|_{0} \leqslant M\left(h^{2}+\tau^{m_{\alpha}}\right), \quad \text { if } 0.5 \leqslant \alpha \leqslant 1 .
$$

To prove Lemma 4 the solution $z_{2}$ of problem (III) has to be evaluated for $\Psi \equiv 0$. We write $v_{s}$ and correspondingly $z_{2}$ in the form

$$
\begin{array}{cl}
v_{s}=v_{s}^{(1)}+v_{s}^{(2)}, & z_{2}=z_{2}^{(1)}+z_{2}^{(2)} \quad \nu_{s}^{(1)}=\tilde{v}_{s}^{(a)}, \\
\bar{v}_{s}=\tilde{v}_{s}^{(0)} h^{2}, & v_{s}^{(2)}=O\left(h \tau^{m_{a}}\right)+O\left(h^{3}\right),
\end{array}
$$


where $\bar{\nu}_{1}^{(0)}$ is determined from formula (2.23) and depends on the values $k, k^{\prime}, k^{\prime \prime}$, $u^{\prime}, u^{\prime \prime}, u^{\prime \prime \prime}, \dot{u}$ when $x=0$ (there is a similar expression for $\left.\tilde{v}_{2}^{(0)}\right)$.

Using Theorem 1 we find

$$
\left\|z_{\mathbf{2}}^{(2)}\right\|_{0} \leqslant M\left(\sqrt{h} \tau^{m_{\alpha}}+h^{\frac{3}{2}}\right)
$$

To evaluate $\left\|z_{2}^{(1)}\right\|_{0}$ we use Theorem 2 , which gives

$$
\begin{aligned}
& \left.\left\|z_{2}^{(1)}\right\|_{0} \leqslant M \max _{0<t^{\prime} \leqslant t}\left[\left|\bar{\nu}_{1}^{(0)}\left(t^{\prime}\right)\right|+\left|\bar{\nu}_{2}^{(0)}\left(t^{\prime}\right)\right|+\left|\left(\bar{v}_{1}^{(0)}\right)-\right|+\mid\left(\bar{v}_{2}^{(0)}\right)\left(t^{\prime}\right)_{t}\right]\right]+ \\
& \left.+\left|\bar{\nu}_{1}^{(0)}(0)\right|+\left|\hat{\nu}_{2}^{(0)}(0)\right|\right\} h^{2} \text {. }
\end{aligned}
$$

Since $\left|\bar{v}_{s}^{(0)}\right|$ and $\left|\left(\bar{v}_{s}^{(0)}\right)_{\bar{t}}\right|$ are bounded it follows that

$$
\|z\|_{2}^{(1)}\left(x, t \|_{0} \leqslant M h^{2}\right.
$$

Using (19) we obtain (18).

4. Accuracy in the class of continuous coefficients

Now let us suppose that the coefficients $k(x, t)$ and $f(x, t, u, p, q)$ do not have lines of discontinuity $\left(v_{0}=0\right)$ and the conditions formulated in section 2 are satisfied over the entire domain $\bar{\mu}$. Then

$$
\Psi=O\left(h^{2}\right)+O\left(\tau^{m_{\alpha}}\right) \text { at all points of the net } \Omega
$$

THEOREM 3. When $h$ and $\tau$ independently tend towards 0 the solution $y$ of problem (II) uniformly converges to the solution $u(x, t)$ of problem (I), so that when $\tau<\tau_{0}$ the following inequality is true

$$
\|y-u\|_{0} \leqslant M\left(h^{2}+\tau^{m_{\alpha}}\right) \quad \text { for } \quad 0.5 \leqslant \alpha \leqslant 1,
$$

if condition (20) is satisfied.

To prove Theorem 3 it is sufficient to evaluate $z_{1}$-the solution of problem (III) when $v_{1} \equiv 0, v_{2} \equiv 0$-from Theorem 1 and then use Lemma 4 .

Noting that $\|\psi(x, t)\|_{2}=O\left(h^{2}\right)+O\left(\tau^{m_{\alpha}}\right)$ and using Theorem 1 , we find

$$
\left\|z_{1}(x, t)\right\|_{0} \leqslant M\left(h^{2}-1 \tau^{m_{\alpha}}\right) \text {. }
$$

5. Approximation error in the neighbourhood of a line of discontinuity

Let the functions $k(x, t)$ and $f(x, t, u, p, q)$ and their derivatives $\frac{\partial f}{\partial u}, \frac{\partial f}{\partial p}$, and $\frac{\partial f}{\partial q}$ have discontinuities of the first kind with respect to $(x, t)$ on a certain curve $x=\eta_{y}(t)$ belonging to the family $\left\{\Gamma_{v}\right\}$ (see $\S 1.2$ ). We shall investigate the asymptotic behaviour of the approximation error $\Psi$ of the scheme $\mathscr{P}_{h r}^{\alpha}$ at the points $\left(x_{n_{v}}, t\right)$ and $\left(x_{n_{p+1}}, t\right)$ of the net $\Omega$.

To simplify the notation we shall omit the index $\nu$ and write $\eta, n, \theta$ instead of $\eta_{v}, n_{v}$ and $\theta_{v}$, setting $\eta=x_{n}+\theta h, x_{n}=n h, 0 \leqslant \theta \leqslant 1$ (see section 2). The net function

$$
\Psi=\left(\psi_{a}+\psi_{p}+\psi_{\varphi}\right)^{(\alpha)}+\psi_{q}
$$

is determined from formulae (1.36)-(1.41). 
The expansion for $\Psi_{a}$ at the points $x=x_{n}$ and $x=x_{n+1}$ was obtained in [1]:

$$
\begin{aligned}
h \psi_{a, n}=O(1), \quad h \psi_{a, n+1} & =O(1), \quad \psi_{a, n}+\psi_{a, n+1}=O(1) \\
\left(\psi_{a, n}\right. & \left.=\psi_{a}\left(x_{n}, t\right)\right)
\end{aligned}
$$

for any scheme of the initial family. For the best canonical scheme (1.28) with the pattern functional

we have

$$
A[\mu(s)]=\left[\int_{-1}^{0} \frac{\mathrm{d} s}{\mu(s)}\right]^{-1}, \quad F[\mu(s)]=\int_{-0.5}^{0.5} \mu(s) \mathrm{d} s
$$

$$
\left.\psi_{a, n}=O(1), \quad \psi_{a, n}+\psi_{a, n+1}=(0 \cdot 5-\theta)\left[k u^{\prime}\right)_{\pi}^{\prime}-\left(k u^{\prime}\right)_{\pi}^{\prime}\right]+O(h) .
$$

Now let us consider the error $\psi_{p}$ at $x=x_{n}$ and $x=x_{n+1}$

$$
\begin{gathered}
\psi_{p, n}=\left(\frac{\partial \vec{\varphi}}{\partial p}\right)_{n}\left(a_{n+1} u_{x, n}+a_{n} u_{\bar{x}, n}-2\left(k u^{\prime}\right)_{n}^{\prime}\right) . \\
\psi_{p, n+1}=\left(\frac{\partial \varphi}{\partial p}\right)_{n+1}\left(a_{n+2} u_{x, n+1}+a_{n+1} u_{x, n}-2\left(k u^{\prime}\right)_{n+1}\right)
\end{gathered}
$$

where $(\partial \varphi / \partial p)_{n}$ and $(\partial \varphi / \partial p)_{n+1}$ are the values of the derivative $\partial \varphi / \partial p$ for certain mean values $\bar{p}_{n}$ and $\bar{p}_{n+1}$ of the argument $p$; thus, for example,

$$
\bar{p}_{n}=2\left(k u^{\prime}\right)_{n}+\theta_{1}\left(a_{n+1} u_{x, n}+a_{n} u_{\bar{x}, n}-2\left(k u^{\prime}\right)_{n}\right), \quad 0 \leqslant \theta_{1} \leqslant 1 .
$$

It follows from the expansions $u_{x, n}=\theta u_{n}^{\prime}+(1-\theta) u_{n}^{\prime}+O(h), u_{\bar{x}, n}=u_{n}^{\prime}+O(h)$ $u_{x, n+1}=u_{n}^{\prime}+O(h), a_{n}=k_{n}+O(h), a_{n+2}=k_{\mathrm{n}}+O(h)$ that:

$$
\begin{gathered}
a_{n+1} u_{x, n}+a_{n} u_{\bar{x}_{x}, n}-2\left(k u^{\prime}\right)_{n}=\left[a_{n+1}\left(\frac{\theta}{k_{\pi}}+\frac{1-\theta}{k_{\text {In }}}\right)-1\right] W+O(h), \\
a_{n+2} u_{x, n+1}+a_{n+1} u_{x, n}-2\left(k u^{\prime}\right)_{n+1}=\left[a_{n+1}\left(\frac{\theta}{k_{\pi}}+\frac{1-\theta}{k_{\text {II }}}\right)-1\right] W+O(h),
\end{gathered}
$$

where $W=k_{\mathrm{n}} u_{\mathrm{s}}^{\prime}=k_{\mathrm{n}} u_{\mathrm{n}}^{\prime}(\operatorname{see}(1.5))$

Hence

$$
\psi_{p, n}=O(1), \quad \psi_{p, n+1}=O(1)
$$

in the initial class of schemes, and for the best canonical scheme

because in this case

$$
\psi_{p, n}=O(h), \quad \psi_{p, n+1}=O(h),
$$

$$
a_{n+1}\left(\frac{\theta}{k_{\mathrm{I}}}+\frac{1-\theta}{k_{\mathrm{II}}}\right)-1=O(h) .
$$

It can be easily seen that the following lemma is true.

Lemma 5. If $\eta=$ const. when $0 \leqslant t \leqslant T$ (fixed discontinuity) and the function $\frac{\partial f^{x-1}}{\partial p^{x-1}}(x, t, u, p, q)$ satisfies Lipshitz's condition for $t, u, p$ and $q$, then

$$
\left|\left(\psi_{p, n}\right)_{\bar{t}}\right| \leqslant M h^{x-1}, \quad\left|\left(\psi_{p, n+1}\right)_{\bar{t}}\right| \leqslant M h^{x-1},
$$

where $x=1$ for the entire class of initial schemes, $x=2$ for the scheme (24). 
We now find out the value of the expression

$$
\psi_{\varphi}=F\left[f\left(x+s h, t, u, 2 k u^{\prime}, \dot{u}\right]-f\left(x, t, u, 2 k u^{\prime} \dot{u}\right) \quad(-0.5 \leqslant s \leqslant 0.5) .\right.
$$

at the points $x=x_{n}$ and $x=x_{n+1}$.

It will be assumed that the discontinuity is fixed, i.e. $\eta=$ const. when $0 \leqslant t \leqslant T$. Because of Note $1(\S 1.1)$ the derivative $u$ is continuous when $x=\eta\left(\dot{u}_{\pi}=\dot{u}_{\Pi}\right)$.

Two cases are possible: $\theta<0.5$ and $\theta>0.5$. We carry out the reasoning assuming that $\theta<0.5$. Since the discontinuity is outside the interval $\left(x_{n}+0.5 h\right.$, $\left.x_{n+1}+0.5 h\right)$, we find directly that $\Psi_{\varphi, n+1}=O(h)^{2}$ for any initial scheme.

Next we consider the best scheme (24), for which

$$
\psi_{\varphi, n}=\int_{-0.5}^{0.5} f\left(x_{n}+s h, t, u_{n}, 2\left(k u^{\prime}\right)_{n}, \dot{u}_{n}\right) \mathrm{d} s-f\left(x_{n}, t, u_{n}, 2\left(k u^{\prime}\right)_{n}, \dot{u}_{n}\right),
$$

and assume that the discontinuity is fixed.

Expanding the function under the integral in the neighbourhood of the point $x=\eta$ we find that:

1) If $s<\theta$, then

$$
\begin{aligned}
& f\left(x_{n}+s h, t, u_{n}, 2\left(k u^{\prime}\right)_{n}, \dot{u}_{n}\right)=f_{\Omega}+\left\{(s-\theta)\left(\frac{\partial f}{\partial x}\right)_{\pi}-\left[\left(\frac{\partial f}{\partial u}\right)_{\pi} u_{I}^{\prime}+\right.\right. \\
& \left.\left.+\left(\frac{\partial f}{\partial p}\right)_{\pi} 2\left(k u^{\prime}\right)_{\pi}^{\prime}+\left(\frac{\partial f}{\partial q}\right)_{\pi}^{\prime} \dot{u}_{\pi}^{\prime}\right] \theta\right\} h+O\left(h^{2}\right) \quad\left(\dot{u}^{\prime}=\frac{\partial^{2} u}{\partial x \partial t}\right)
\end{aligned}
$$

2) If $s>0$, then

$$
\begin{gathered}
f\left(x_{n}+s h, t, u_{n}, 2\left(k u^{\prime}\right)_{n}, \dot{u}_{n}\right)=f_{\mathrm{n}}+\left\{(s-\theta)\left(\frac{\partial t}{\partial u}\right)_{\mathrm{n}}-\left[\left(\frac{\partial f}{\partial u}\right)_{\mathrm{n}} u_{\pi}^{\prime}+\left(\frac{\partial f}{\partial p}\right)_{\mathrm{n}} 2\left(k u^{\prime}\right)_{\pi}^{\prime}+\right.\right. \\
\left.\left.+\left(\frac{\partial f}{\partial q}\right)_{\mathrm{n}} \dot{u}_{n}^{\prime}\right] \theta\right\} h+O\left(h^{2}\right)=f_{\mathrm{n}}+h(s-\theta)\left(\frac{\partial f}{\partial x}\right)_{\mathrm{n}}+\beta h+O\left(h^{2}\right) .
\end{gathered}
$$

The expansion for the second term in (29) is obtained from formula (30) when $s=0$. Substituting these expressions in (29) we find

$$
\begin{aligned}
\psi_{\phi_{x} n}= & (0.5-\theta)\left(f_{\mathrm{n}}-f_{n}\right)+ \\
& +0.5(0.5-\theta)\left[(0.5+\theta)\left(\frac{\partial f}{\partial x}\right)_{\pi}+(0.5-\theta)\left(\frac{\partial f}{\partial x}\right)_{n}+2 \beta\right] h+O(h) .
\end{aligned}
$$

To expand $\psi_{\varphi, n}$ for an arbitrary scheme of the initial family we use formulae (30) and (31), writing the principal terms only:

where

$$
f\left(x_{n}+s h, t, u_{n} .2\left(k u^{\prime}\right)_{n}, \dot{u}_{n}\right)=f^{*}(s)+O(h),
$$

Then

$$
f^{*}(s)= \begin{cases}f_{n} & s<\theta \\ f_{n} & s>\theta\end{cases}
$$

$$
\psi_{\varphi, n}=F\left[f^{*}(s)\right]-f_{\Omega}+O(h)=O(1) \text {. }
$$


Collecting all the results we obtain the following expansion in powers of $h$ for $\psi_{n}$ and $\psi_{n+1}$ :

$$
\begin{gathered}
\psi_{n}=\left(\frac{\beta-1}{h}+\beta_{0}+\beta_{1} h\right)+O\left(h^{2}\right)=\bar{\psi}_{n}+O\left(h^{2}\right) \quad\left(\psi=\psi_{a}+\psi_{p}+\psi_{\varphi}\right), \\
\psi_{n}+\psi_{n+1}=\left(\gamma_{0}+\gamma_{1} h\right)+O\left(h^{2}\right)=\left(\bar{\psi}_{n}+\bar{\psi}_{n+1}\right)+O\left(h^{2}\right),
\end{gathered}
$$

where $\beta_{-1}$ depends on the limiting values of the function $k, u^{\prime}$ of the left and right on the lines $x=\eta ; \beta_{0}$ and $\gamma_{0}$ depend on the limiting values of $k, k^{\prime}, u^{\prime}, u^{\prime \prime}, \beta_{1} ; \gamma_{1}$ depends on the limiting values of $k, k^{\prime}, k^{\prime \prime}, u^{\prime}, u^{\prime \prime}, u^{\prime \prime \prime}, \dot{u}^{\prime}$.

$\beta_{-1}, \beta_{0}, \gamma_{0}, \beta_{1}$ and $\gamma_{1}$ satisfy Lipshitz's conditions for all the arguments. For the best canonical scheme (24)

$$
\beta_{1}=0, \quad \gamma_{0}=(0.5-\theta)\left[\left(\mathcal{P}_{u}\right)_{\mathrm{n}}-(\mathcal{P} u)_{\pi}\right]=0 .
$$

the following lemma is then proved automatically.

Lemma 6. If $k(x, t)$ and $f(x, t, u, p, q)$ have discontinuities of the first kind on the straight line $x=\eta=$ const. and all the conditions mentioned above (in sections 2 and 5 ) are fulfilled then

$$
\left.\begin{array}{ll}
h \bar{\psi}_{n}=O\left(h^{x-1}\right), & \bar{\psi}_{n}+\bar{\psi}_{n+1}=O\left(h^{x-1}\right), \\
h\left(\bar{\psi}_{n}\right)_{\bar{t}}=O\left(h^{x-1}\right), & \left(\bar{\psi}_{n}+\bar{\psi}_{n+1}\right)_{\bar{t}}=O\left(h^{x-1}\right),
\end{array}\right\}
$$

where $x=1$ for the entire class of initial schemes, $x=2$ for the scheme (24).

Since $\dot{u}$ is continuous for $x=\eta$ in the case of a fixed discontinuity,

$$
\psi_{q}=O\left(\tau^{m_{\alpha}}\right) \quad \text { at all points of the net } \Omega
$$

If the discontinuity is moving, then $\dot{u}_{\mathrm{n}} \neq \dot{u}_{\mathrm{m}}$ and $\gamma_{0} \neq 0$ for scheme (24) also (condition $\beta_{-1}=0$ for scheme (24) is always satisfied).

Note. As the above arguments show, if a line of discontinuity passes through the points $\left(x_{n}, t\right)$ or $\left(x_{n+1}, t\right)$ of the net $\Omega(\theta=0$ or $\theta=1)$ and the function $f(x, t, u, p, q)$ is continuous when $x=\eta$ then for any initial scheme

$$
\bar{\psi}_{n}=O(h), \quad \bar{\psi}_{n+1}=O(h) .
$$

If however the point $x=\eta$ is irrational, then $\theta$ must also be irrational, i.e. in this case the condition $\theta=0$ or $\theta=1$ cannot be realized for any $h=1 / N$.

\section{Accuracy in the class of coefficients having fixed discontinuities}

We shall now evaluate the order of accuracy of the difference problem (II), assuming that $k(x, t)$ and $f(x, t, u, p, q)$ have discontinuities of the first kind on the straight lines $x=\eta_{v}=$ const. $\left(y=1,2, \ldots, v_{0}\right), 0 \leqslant t \leqslant T$. Here $\Psi$ must be written as a sum

where

$$
\Psi=\Psi_{1}+\Psi_{2}
$$

$$
\Psi_{1}=\sum_{v=0}^{v_{0}}\left(\psi_{v}^{*}\right)^{(\alpha)}, \quad \psi_{v}^{*}=\bar{\psi}_{n_{v}} \cdot \delta\left(x, x_{n_{p}}\right)+\bar{\psi}_{n_{p}+1} \delta\left(x, x_{n_{v}+1}\right),
$$

$\delta(x, \xi)$ is Kronecker's symbol $(\delta(x, \xi)=0$ when $x \neq \xi, \delta(\xi, \xi)=1)$. 
The following expression is true for the function $\psi_{2}$ at all points of the net $\Omega$

$$
\left.\Psi_{z}=O\left(h^{2}\right)+O(\tau)^{m_{x}}\right)
$$

Expressions for $\bar{\psi}_{n_{y}}$ and $\bar{\psi}_{n_{v+1}}$ are obtained from formulae (35) and (36).

Since the effect of boundary conditions has been taken into account by Lemma 4 , it remains to find an expression for the function $z_{1}$ which is a solution of the problem

$$
\left.\begin{array}{l}
\left(a z_{\bar{x}}\right)_{x}^{(a)}+Q(z)-\varrho z_{\bar{t}}=-\Psi, \\
l_{1} z=0 \text { for } x=0, \quad l_{2} z=0 \text { for } x=1, \quad z=0 \text { for } t=0 .
\end{array}\right\}
$$

In accordance with (39) we write

$$
z_{1}=z_{11}+z_{12}
$$

where $z_{11}$ is a solution of the problem (42) with the right-hand side of $\Psi_{1}$, and $z_{12}$ a solution with the right-hand side of $\Psi_{2}$. Noting that $\left\|\Psi_{2}\right\|_{2}=O(h)^{2}+O\left(\tau^{m_{\alpha}}\right)$ and using Theorem 1, we find

$$
\left\|z_{12}\right\|_{0} \leqslant M\left(h^{2}+\tau^{m_{a}}\right) .
$$

To estimate $z_{11}$ we need

LeMMA 7. Let $z^{*}$ be a solution of problem (42), where

$$
\Psi=\left(\psi^{*}\right)^{(x)}, \quad \psi^{*}=\bar{\psi}_{n} \delta\left(x, x_{n}\right)+\psi_{n+1} \delta\left(x, x_{n+1}\right) .
$$

then if $\tau<\tau_{0}$ is sufficiently small we have the inequality

$$
\begin{aligned}
\left\|z^{*}(x, t)\right\|_{0} \leqslant M\left\{h^{\frac{3}{2}}\left|\bar{\psi}_{n}(0)\right|+h\left|\bar{\psi}_{n}(0)+\bar{\psi}_{n+1}(0)\right|+\right. & \\
& +\max _{0<t^{\prime} \leqslant t}\left[h^{2}\left(\bar{\psi}_{n}\left(t^{\prime}\right)|+|\left(\bar{\psi}_{n}\left(t^{\prime}\right)\right)_{i}\right)+h\left(\left|\bar{\psi}_{n}\left(t^{\prime}\right)+\bar{\psi}_{n+1}\left(t^{\prime}\right)\right|\right)+\right. \\
& \left.\left.+\left|\left(\bar{\psi}_{n}\left(t^{\prime}\right)\right)_{i}+\left(\bar{\psi}_{n+1}\left(t^{\prime}\right)\right)_{\bar{i}}\right|\right]\right\}+\bar{M}^{\frac{3}{2}} \max _{0<t^{\prime} \leqslant t}\left|\bar{\psi}_{n}\left(t^{\prime}\right)\right|,
\end{aligned}
$$

where $\bar{M}=0$ when $b_{s k}=0, s=1,2 ; k=1,2$.

To evaluate $\left\|z^{*}(x, t)\right\|_{0}$ we need inequality (12) of Theorem 2 containing $\left\|\Psi^{*}\right\|_{i}$, $\left\|\psi^{*}\right\|_{4^{*}},\left\|\psi_{t}^{*}\right\|_{4^{*}}$. Let us calculate these quantities. We first evaluate the function

$$
\mu(x, t)=\sum_{x^{\prime}=h}^{x^{\prime}=x} h \psi^{*}\left(x^{\prime}, t\right) \text {. }
$$

Substituting expression (44) for $\psi^{*}$ we find

$$
\mu(x, t)=\left\{\begin{array}{cc}
0, & x<x_{n}, \\
h \bar{\psi}_{n}, & x=x_{n}, \\
h\left(\bar{\psi}_{n}+\bar{\psi}_{n+1}\right), & x>x_{n} .
\end{array}\right.
$$

In particular, $\mu\left(x_{N-1}, t\right)=h\left(\bar{\psi}_{n}+\bar{\psi}_{n+1}\right)$. Therefore

Using (46) we find

$$
\left|\left(\psi^{*}, 1\right)\right|=h\left|\bar{\psi}_{n}+\bar{\psi}_{n+1}\right| \text {. }
$$

$$
\left\|\psi^{*}\right\|_{1}=\|\mu\|_{2}+\left|\left(\psi^{*}, 1\right)\right| \leqslant h^{\frac{3}{2}}\left|\bar{\psi}_{n}\right|+2\left|\bar{\psi}_{n}+\bar{\psi}_{n+1}\right| h,
$$




$$
\begin{gathered}
\left\|\psi^{*}\right\|_{\mathbf{4}^{*}}=\|\mu\|_{1}+\left|\left(\psi^{*}, 1\right)\right| \leqslant h^{2}\left|\bar{\psi}_{n}\right|+2\left|\bar{\psi}_{n}+\bar{\psi}_{n+1}\right| h, \\
\left\|\psi_{\psi^{*}}^{*}\right\|_{\mathbf{4}^{*}} \leqslant h^{2}\left|\left(\bar{\psi}_{n}\right)_{\bar{t}}\right|+2 h\left|\left(\bar{\psi}_{n}+\bar{\psi}_{n+1}\right)_{\bar{t}}\right| .
\end{gathered}
$$

(45) follows from this and inequality (12).

Because of Lemma 7 we have for $z_{11}$

and consequently

$$
\left\|z_{11}(x, t)\right\|_{0} \leqslant M h^{x-\frac{1}{2}}
$$

$$
\left\|z_{1}(x, t)\right\|_{0} \leqslant M\left(h^{x-\frac{1}{2}}+\tau^{m_{\alpha}}\right) .
$$

This result is used to prove the following theorem.

THEOREM 4. If $k(x, t)$ and $f(x, t, u, p, q)$ have discontinuities of the first kind on a finite number of straight lines $x=\eta_{v}=$ const., $\nu=1,2, \ldots, v_{0}$ and all the conditions mentioned above (in sections 2 and 5) are satisfied, then the solution $y(x, t)$ of the difference problem (II) converges uniformly to the solution $u(x, t)$ of problem (I) when $h$ and $\tau$ tend independently towards 0 . Thus for any scheme $\mathscr{P}_{h \tau}^{x}$ of the initial family the following expression is true when $\tau<\tau_{0}$ is sufficiently small

$$
\|y-u\|_{0} \leqslant M\left(h^{\frac{1}{2}}+\tau^{m_{x}}\right) \text { for } 0.5 \leqslant \alpha \leqslant 1
$$

and for the best canonical scheme (24)

$$
\|y-u\|_{0} \leqslant M\left(h^{\frac{3}{2}}+\tau^{m_{\alpha}}\right) \text {. }
$$

To prove this theorem it is sufficient to evaluate the function $z_{1}(x, t)$ from section 3 and use Lemma 4.

\section{REMARKS}

1. The solution of problem (II) for an arbitrary difference scheme $\mathscr{P}_{h r}^{\alpha}$ of the initial family in a class of discontinuous coefficients $k(x, t)$ converges under much less rigorous conditions: 1) $k, k^{\prime}, f(x, t, u, p, q), u^{\prime \prime}, \dot{u}$ are continuous in $\bar{\Delta}_{v}(v$ $\left.\left.=0,1, \ldots, v_{0}\right), 2\right) k$, $u^{\prime}$ satisfy Lipshitz's condition for $t$ along $\left.\Gamma_{v}, 3\right) k(x, t)$ in $\bar{\Delta}_{v}$ and $\sigma_{s}(t)$ when $0 \leqslant t<T$ satisfy Lipshitz's condition for $t$. Under these conditions

$$
\|y+u\|_{0} \leqslant \varrho_{0}(h)+\underline{o}_{0}(\tau)
$$

where $\varrho_{0}(h) \rightarrow 0$ when $h \rightarrow 0$ and $\varrho_{0}(\tau) \rightarrow 0$ when $\tau \rightarrow 0$.

2. To simplify the treatment only the conditions sufficient for ensuring the expressions obtained above are formulated. Some of the requirements imposed on these solutions $u(x, t)$ and the coefficients of problem (I) can be relaxed. Thus, for example, Theorem 4 remains valid without the requirement that the limiting values of the functions $k^{\prime \prime}, u^{\prime \prime \prime}, u^{\prime}$ on the straight lines $I_{v}^{\prime}\left(v=1,2, \ldots, v_{0}\right)$ should satisfy Lipshitz's condition for $t$.

3. If $\frac{\partial f}{\partial p}(x, t, u, p, q)=0$ and one of the following conditions is satisfied: 1$) u(x, 0)=\dot{u}(x, 0)$ 
$=0.2)$ The heat-conduction coefficient $k$ is independent of $t$, then instead of (50)-(51) the following inequality

$$
\|y-u\|_{0} \leqslant M\left(h^{*}+\tau^{m_{\alpha}}\right),
$$

is satisfied, i.e. in the class of discontinuous coefficients scheme (24) has the same order of accuracy as in the class of small coefficients $k(x, t)$ and $f(x, t, u, p, q)$ of the equation $\mathscr{P}_{u}=0$.

4. Theorem 4 is also true for the first boundary problem $\left(u(0, t)=u_{1}(t), u(1, t)=u_{2}(t)\right)$. In particular, the corresponding results of [3] for the linear equation of heat-conduction follow from this. For a four-point scheme $\mathscr{P}_{h r}^{(1)}(\alpha=1)$ Theorem 4 can be made more rigorous by using special $a$ priori estimates which will be considered separately.

5. Differential difference equations obtained by Rothe's method and the method of straight lines are investigated similarly.

In conclusion the author takes this opportunity to express his gratitude to A. N. Tikhonov for discussing the results obtained.

Translated by PRASENITT Basu

\section{REFERENCES}

1. TIKHONOV, A. N. and SAMARSKII, A. A., Zh. vych. mat. 1: No. 1, 4-63, 1961.

2. SAMARSKII, A. A., Proceedings of the All-Union Conference on differential equations (Erevan, 1958). Armenian Acad. Sci. 1960, 148-60.

3. SAMARSKI, A. A. and FRYAZINOV, F. V., Zh. vych. mat. 1: No. 5, 806-24, 1961 .

4. SAMARSKII, A. A., Zh. vych. mat. 1 : No. 3, 441-60, 1961.

5. SAMARSKII, A. A., Zh. vych. mat. 1: No. 6, 971-1000, 1961.

6. DOUGLAS, J., On the numerical integration of quasi-linear parabolic differential equations. Pacif. J. Math. 6: No. 1: 35-42, 1956.

7. DOUGLAS, J., The application of stability in the numerical solution of quasi-linear parabolic differential equations. Trans. Amer. Math. Soc. 89 : No. 2, 484-518, 1958.

8. DU FORT, E. C. and FRANKEL, S. P., Stability condition in the numerical treatment of parabolic differential equations. Math. Tables and Other Aids Comput. 7: No. 43, 135-52, 1953.

9. ROSE, M., On the integration of non-linear parabolic equations by implicit difference methods. Quart. Appl. Math. 14: No. 3, 237-48, 1956.

10. LEES, M., Approximate solutions of parabolic equations. J. Soc. Industr. and Appl. Math. 7: No. 2, 167-83, 1959.

11. SAUL'EV, V.K., Integrirovanie uravnenii parabolicheskogo tipa metodom setok. (Integration of equations of parabolic type by the method of net.) Moscow-Leningrad, Fizmatgiz, 1960.

12. SAMARSKU, A. A., Dokl. Akad, Nauk SSSR, 121 : No. 2, 225-8, 1958.

13. OLEINIK, O. A., Izv, Akad. Nauk SSSR, ser. mat. 25: No. 1, 3-20, 1961.

14. KAMYNIN, L. I. and MASLENNIKOVA, V. N., Dokl. Akad. Nauk SSSR 133 : No. 5, 1003-6, 1960.

15. KAMYNIN, L. I., Dokl. Akad. Nauk SSSR 139: No. 5, 1048-51, 1961.

16. TIKHONOV, A.N. and SAMARSKII, A. A., Dokl. Akad. Nauk SSSR 124: No. 4, 779-82, 1959.

17. MARCHUK, G.I., Chislennye metody rascheta yadernykh reaktorov. (Numerical methods for designing nuclear reactors.) Moscow, Atomizdat, 1958.

18. BEREZIN, I. S. and ZHIDKOV, N.P., Metody vychislenii. Computing methods) Vol. II, Moscow, Fizmatgiz, 1959.

19. LOKUTSIEVSKII, O. V., Usp. mat. nauk 11: No. 3(69), 2 24, 1956. 